\title{
Modes of synchronisation in the wake of a streamwise oscillatory cylinder
}

\author{
Guoqiang Tang ${ }^{1}$, Liang Cheng ${ }^{1,2,} \uparrow$, Feifei Tong ${ }^{2,}$, Lin $\mathrm{Lu}^{1}$ and Ming Zhao ${ }^{3}$ \\ ${ }^{1}$ DUT-UWA Joint Research Centre, State Key Laboratory of Coastal and Offshore Engineering, \\ Dalian University of Technology, No. 2 Linggong Road, Dalian 116024, China \\ ${ }^{2}$ School of Civil, Environmental and Mining Engineering, The University of Western Australia, 35 \\ Stirling Highway, Perth, WA 6009, Australia \\ ${ }^{3}$ School of Computing, Engineering and Mathematics, Western Sydney University, Locked Bay 1797 , \\ Penrith, NSW 2751, Australia
}

(Received $\mathrm{xx}$; revised $\mathrm{xx}$; accepted $\mathrm{xx}$ )

A numerical analysis of flow around a circular cylinder oscillating in-line with a steady flow is carried out over a range of driving frequencies $\left(f_{d}\right)$ at relatively low amplitudes $(A)$ and a constant Reynolds number of 175 (based on the free-stream velocity). The vortex shedding is investigated, especially when the shedding frequency $\left(f_{s}\right)$ synchronises with the driving frequency. A series of modes of synchronisation are presented, which are referred to as the $p / q$ modes, where $p$ and $q$ are natural numbers. When a $p / q$ mode occurs, $f_{s}$ is detuned to $(p / q) f_{d}$, representing the shedding of $p$ pairs of vortices over $q$ cycles of cylinder oscillation. The $p / q$ modes are further characterised by the periodicity of the transverse force over every $q$ cycles of oscillation, and a spatial-temporal symmetry possessed by the global wake. The synchronisation modes $(p / q)$ with relatively small natural numbers are less sensitive to the change of external control parameters than those with large natural numbers, while the latter is featured with a narrow space of occurrence. Although the mode of synchronisation can be almost any rational ratio (as shown for $p$ and $q$ smaller than 10), the probability of occurrence of synchronisation modes with $q$ being an even number is much higher than $q$ being an odd number, which is believed to be influenced by the natural even distribution of vortices in the wake of a stationary cylinder.

Key words: flow-structure interactions, vortex dynamics, vortex streets

\section{Introduction and motivation}

The frequency of vortex shedding $\left(f_{s}\right)$ from a forced-excitation body may undergo a departure from the inherent Strouhal number $\left(f_{S t}\right)$ of the otherwise stationary body, as a result of the interaction between the self-excited wake instability and the externally imposed perturbation (Konstantinidis $\&$ Balabani 2007). Such a feature has been used as a method in active wake control (Karniadakis \& Triantafyllou 1989; Griffin \& Hall 1991). At certain combinations of driving frequency $\left(f_{d}\right)$ and amplitude $(A)$ of harmonically perturbed oscillations, the vortex shedding may synchronise with the cylinder oscillation, where the flow is characterised by ordered and repeatable wake formation.

Some distinct modes of synchronisation have been observed in the wake of a controlledoscillatory circular cylinder over a range of Reynolds numbers, $\operatorname{Re}=U_{\infty} D / v$, where $U_{\infty}$ is the free stream velocity, $D$ is the cylinder diameter, and $v$ is the kinematic viscosity. Published works in this respect include a cylinder undergoing one-degree-of-freedom (1-dof) sinusoidal vibration either in the transverse direction to the incident flow (Bishop \& Hassan 1964; Koopmann 1967; Griffin \& Ramberg 1974; Stansby 1976; Williamson \& Roshko 1988; Ongoren \& Rockwell 1988a; 
Anagnostopoulos 2000; Carberry, Sheridan \& Rockwell 2005; Leontini et al. 2006; Morse \& Williamson 2009), or in the incident flow direction (Tanida, Okajima \& Watanabe 1973; Griffin \& Ramberg 1976; Sarpkaya, Bakmis \& Storm 1984; Mittal et al. 1991; Zdravkovich 1996; Cetiner \& Rockwell 2001; Xu, Zhou \& Wang 2006; Al-Mdallal, Lawrence \& Kocabiyik 2007; Marzouk \& Nayfeh 2009). Commonly, $f_{s} / f_{d}$ synchronises to $N$ or $1 / N$ (where $N$ is an integer number) when $f_{d}$ is respectively close to the superharmonics $\left(N f_{S t}\right)$ or subharmonics $\left(f_{S t} / N\right)$ of the Strouhal number (Ongoren \& Rockwell 1988a; Konstantinidis \& Bouris 2016). The majority of the aforementioned research focused on synchronisations with $N=1$ for a transversely oscillatory cylinder and $N=2$ for an in-line oscillatory cylinder, which is normally referred to as the primary synchronisation or 'lockon'. Although this synchronisation phenomenon is best known for the translational oscillation, similar wake synchronisation has also been observed for rotatory oscillation circular cylinders (Baek \& Sung 2000; D'Adamo, Godoy-Diana \& Wesfreid 2011) or the combined movements (Nazarinia et al. 2012).

Modes of synchronisation other than $N$ or $1 / N$ have also been reported in the perturbed wake of a circular cylinder. For a transversely oscillatory cylinder, Ongoren \& Rockwell (1988a) found the frequency of recovered vortices synchronised to $2 / 3$ (or $1 / 1.5$ in their content) of the driving frequency (i.e., $f_{s} / f_{d}=2 / 3$ ). Similar to other modes in the work $(1 / N)$, the $2 / 3$ mode was attributed to the nonlinear interaction between the motion of the cylinder and the wake, which gives rise to a number of sum and difference of $f_{s}$ and $f_{d}$ in the spectra. For the flow around an in-line oscillatory circular cylinder, in addition to the 'quasi-lock-on' and 'intermittent lock-on' flow features over a wide range of controlling parameters, a 'six-fold' change in $f_{s} / f_{d}=0.5,1,1.5,2,2.5,3$ was experimentally observed when $f_{d}$ was varied in the range of approximately $f_{d} / f_{S t}=0.5 \sim 2.5$ by Cetiner \& Rockwell (2001). It is noted that this would have had introduced more types of synchronisation frequency ratios $\left(f_{s} / f_{d}\right)$ at relatively high Reynolds numbers (405 2482).

Detemple-Laake \& Eckelmann (1989) carried out an experimental study on the $f_{s}$ and vortex formation from a circular cylinder under the influence of sound waves, which was superimposed on the flow in-line with the free stream. With remarkable analogies to the wake of a translationaloscillatory cylinder in a steady flow, the Kármán vortices were found to shed at the sound frequency or at sub-harmonics of the sound frequency and the flow phenomenon was observed to be independent of the Reynolds numbers in the range from 50 to 250. Although the most stable mode of shedding locked-on to half of the sound frequency, Detemple-Laake \& Eckelmann (1989) also reported that, at certain conditions, $f_{s}$ was pulled to rational ratios of $2 / 3,3 / 4$ and 4/7 of the sound frequency. This is one of the first studies that reported these mode ratios, although the perturbation was due to the external sound waves.

Baek \& Sung (2000) numerically investigated vortex shedding around a circular cylinder undergoing rotational oscillations to quantify quasi-periodicity in the wake. In a few cases when the forced oscillation frequency $\left(S_{f}\right)$ is close to the natural vortex-shedding frequency, the wake was found to be approximately but not "exactly recovered" after a certain number of cycles of oscillation, such as that $S_{t 0} / S_{f} \approx 12 / 11$, where $S_{t 0}$ is the recovered shedding frequency. In other words, approximately 12 vortex pairs shed during 11 forcing periods. Baek \& Sung (2000) further proposed that an irrational $S_{t 0} / S_{f}$ leads to a quasi-periodic flow, while a rational $S_{t 0} / S_{f}=p / q$ gives rise to a periodic flow ( $p$ and $q$ are integer numbers). In addition, nonlinear oscillation models were successfully applied in analysing the frequency components. However, possibly due to the focus being on quasi-periodic flows, the study did not provide examples of synchronisation states other than $p / q=1$.

More recently, nonlinear synchronisations or $P_{N}$ modes for an in-line oscillatory cylinder at $R e \leq$ 
250 have been reported (Leontini, Lo Jacono \& Thompson $2011 ; 2013$ ), where $f_{s} / f_{d}$ synchronises to rational ratios in the form of $(N-1) / N$ and integers $N$ as large as 8 were observed (i.e., the $P_{8}$ mode). It was noted that the $1 / 2$ mode and 2/3 mode reported by Ongoren $\&$ Rockwell (1988a) are just special cases in a series of rational-mode ratios, $(N-1) / N$. Leontini et al. $(2011 ; 2013)$ also offered an informative discussion on the wake frequency selection at a range of fixed $f_{d}$ and $R e$. The $f_{s}$ was found to be a continuous function of the squared oscillation amplitude $(A / D)$, with discontinuous steps overlaid on the trend due to the synchronised $P_{N}$ modes, which was latter experimentally reproduced at high $R e \in$ [3750-6300] (Tudball-Smith et al. 2012). The modes with a numerator other than $N-1$ were not reported in their studies.

A popular physical explanation on the frequency content in the near-wake flow has been identified as the competition between vortex formation induced by the steady flow and the cylinder oscillation. $\mathrm{Xu}$ et al. (2006) provided a discussion on the mode competition through a dimensional analysis. Although from all interpretations on the synchronisation phenomenon, there is no restriction for $f_{s} / f_{d}$ being synchronised to other rational ratios (for example 3/5), investigations in this respect are limited. Exceptions include Olinger \& Sreenivasan (1988), Olinger (1993) and Olinger (1998) who demonstrated through experiments on a transversely oscillatory cylinder in air at $R e \approx 55$, that the wake frequencies are "lock-in" to approximately 30 rational ratios of $f_{d}$. Rational ratios, such as $3 / 5$ and 2/7, are not uncommon in their work and occupy reasonable widths over the external frequency. Over a range of relatively large $R e$ between 420-435, Woo (1999) observed approximately ten more phase-locked states in a brief note. Both Olinger \& Sreenivasan (1988) and Woo (1999) correlate these phase-locked phenomena in fluid dynamics to the resonant responses of nonlinear dynamical systems with coupled oscillators or oscillators coupled to periodic external excitations. Woo (1999) even implied that any reasonably fine phase-locked state could be detected given properly controlled cylinder oscillation in the $\left(f_{d} / f_{S t}, A / D\right)$-plane. However, relatively less attention has been paid to this multiplicity of synchronisation states by subsequent studies.

This work is motivated by the abundance of the synchronisation modes and attempts to obtain further understanding on how the flow is synchronised with the external perturbation in the wake of an in-line oscillatory cylinder in steady flow. A variety of subharmonic synchronisation modes, identified for the case of a transversely oscillating cylinder (Olinger \& Sreenivasan 1988; Woo 1999), is shown to exist for the case of an in-line oscillating cylinder. These modes of synchronisation are referred to as $p / q$ modes, where $p$ and $q$ are natural numbers (taken as $0,1,2,3$, $4, \ldots)$ and the mode ratio $p / q$ can potentially be any rational ratio between $[0,1]$ in the subharmonics regime. From the common feature of perturbed wakes as discussed above, it is anticipated that the main findings from the present study should also apply to other types of externally imposed perturbation, such as transverse or rotatory oscillations.

The remainder of this paper is organised as follows. Numerical methods and model validations are introduced in $\S 2$. The main findings are presented in $\$ 3$ with a demonstration on how the modes of synchronisation are identified through the time representation of transverse forces, followed by a presentation of a synchronisation map of the identified modes in the $\left(f_{d} / f_{S t}, A / D\right)$-plane and selected flow features in several modes. A complete series of modes of synchronisation is further discussed in $\S 4$, along with the mode selection of a synchronised flow. Finally, the concluding remarks are presented in $\S 5$. 


\section{Methodology}

\section{1. $\quad$ Numerical method}

The study is carried out numerically based on a two-dimensional numerical model at $R e=175$, following Leontini et al. (2011; 2013), who have also demonstrated the generality of the results over $R e=75 \sim 250$ by two-dimensional simulations. Although the possibility of the flow becoming threedimensional cannot be ruled out over the parameter space covered in this study, the threedimensionality is expected to be weak and has a limited effect on the characteristics of flow. The consistency among experimental studies by Olinger \& Sreenivasan (1988), Woo (1999) and TudballSmith et al. (2012) at different flow regimes as discussed earlier, provides further confidence on this. The governing equations for the flow are the incompressible Navier-Stokes (NS) equations,

$$
\nabla \cdot \boldsymbol{U}=0,
$$

$$
\frac{\partial \boldsymbol{U}}{\partial t}+\boldsymbol{U} \cdot \nabla \boldsymbol{U}=-\nabla \beta \gamma+\frac{1}{R e} \nabla^{2} \boldsymbol{U}+\boldsymbol{a},
$$

where $\boldsymbol{U}=(u, v)$ is the velocity in the $x$ - and $y$-directions, $t$ is the time and $\beta c$ is the kinematic pressure. Direct numerical simulations are carried out by using an open-source Spectral/hp element code, Nektar++ (Cantwell et al. 2015). The code employs high-order quadrilateral expansions within each element through the Gauss-Lobatto-Legendre quadrature points $\left(N_{p}\right)$. A second-order implicitexplicit time-integration scheme is chosen from the embedded incompressible NS solver, along with the velocity correction splitting scheme and a continuous Galerkin projection.

Herein the harmonic cylinder oscillation is implemented through a moving frame fixed on the cylinder by introducing a forcing term, $\boldsymbol{a}$, which is the additional acceleration as the result of the non-inertial translation of the reference frame as detailed by Newman \& Karniadakis (1997). This technique avoids the difficulty with mesh deformations and has been implemented successfully in various studies (Newman \& Karniadakis 1997; Blackburn \& Henderson 1999; Leontini et al. 2006).
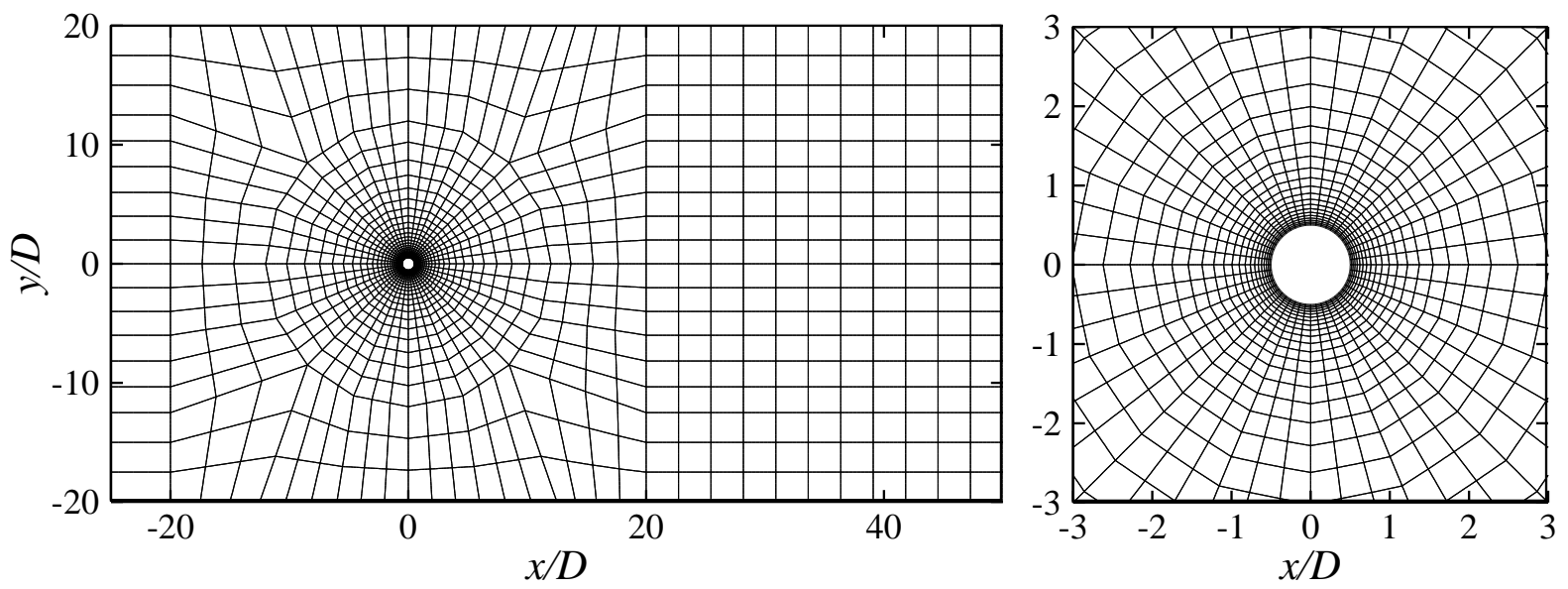

Figure 1 Definition sketch of the computational domain and mesh near the cylinder surface.

Although it is an unbounded flow, a rectangular computational domain is employed in the numerical simulation as shown in Figure 1. At the left, top and bottom boundaries, the Dirichlet boundary conditions of $u=U_{\infty}-u_{c y l}$ and $v=0$ are applied, where $u_{c y l}$ is the forcing cylinder velocity. On the cylinder surface, the no-slip condition is imposed. A zero normal velocity gradient is applied on the outlet boundary. The pressure is fixed to zero at the outlet, while higher-order boundary 
conditions of pressure gradient are implemented on the cylinder surface and the far-field boundaries (Karniadakis, Israeli \& Orszag 1991). In the simulations, the initial values of flow velocity and pressure were set to zero, while the time-step was chosen to keep the Courant number below 1. The independence of the flow fields from the time-step was also checked at a few selected cases.

The displacement of the circular cylinder in the free stream is formulated in the $x$-direction as,

$$
X(\tau)=A / D \sin \left(2 \pi f_{d} \tau\right)
$$

where $A$ is the amplitude of the cylinder oscillation, $f_{d}$ is the non-dimensional driving frequency of the oscillation and $\tau$ is the non-dimensional time (both have been non-dimensionalised by the time scale $\left.D / U_{\infty}\right)$. It is noted that the problem of the steady flow around an oscillatory cylinder is kinematically equivalent to that of non-zero mean oscillatory flow around a fixed cylinder, provided that a transformation in the control variables is carried out (Konstantinidis \& Bouris 2016). In the present study, we have simulated both problems at certain equivalent parameters and the results are identical.

Polynomial order

$\begin{array}{ll}5 & 1.33632 \\ 6 & 1.33626 \\ 7 & 1.33627 \\ 8 & 1.33633 \\ 9 & 1.33625\end{array}$

$\bar{C}_{D}$

1.33632
$f_{S t}$

0.19172

0.19172

0.19172

0.19172

0.19172
$C_{L-r m s}$

0.42835

0.42831

0.42830

0.42831

0.42834

Table $1 N_{p}$-convergence tests on a stationary cylinder for the mean drag coefficient $\left(\bar{C}_{D}\right)$, root-mean-square lift coefficients $\left(C_{L-r m s}\right)$ and the Strouhal number $\left(f_{S t}\right)$.

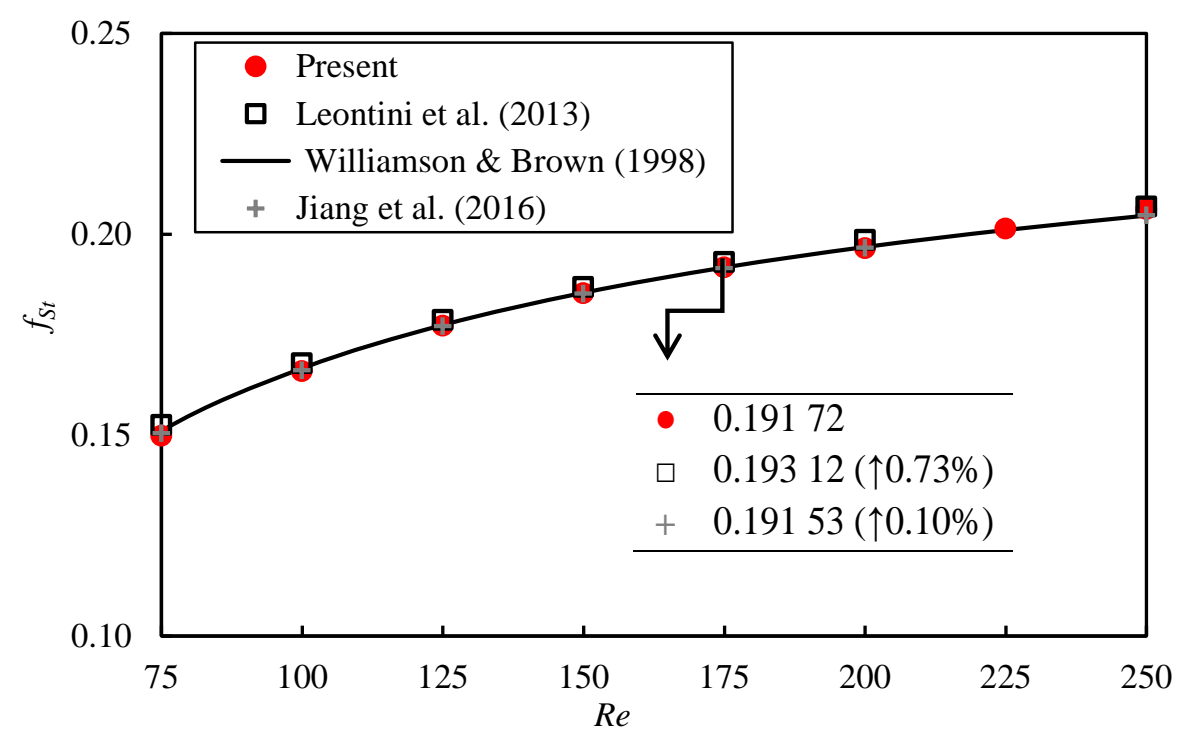

Figure 2 Comparison on Strouhal numbers $\left(f_{S t}\right)$ for a stationary cylinder in steady flow at various Reynolds numbers. The insert box shows those at $R e=175$ and the difference of the present result with the published sources.

\subsection{Model validation}

A computational domain size of $40 D \times 75 D$ with 1806 quadrilateral spectral elements is chosen for the numerical validation on flow around a stationary cylinder. Figure 1 shows the mesh distribution in the whole computational domain along with a close view around the cylinder. The quadrilateral expansion is employed through the Gauss-Lobatto-Legendre quadrature points within each element. In the model validation, five different orders of Lagrange polynomial expansion $\left(N_{p}\right)$ are tested. 
Table 1 provides a summary of the convergent study at $R e=175$ for a stationary cylinder. In this study, $N_{p}$ varies from 5 to 9 , resulting a global number of points ranging from $1806 \times 5^{2}$ to $1806 \times$ $9^{2}$. The converged force coefficients and vortex shedding frequencies in Table 1 show that the flow is generally independent of the chosen $N_{p}$. Here, $C_{D}=F_{x} /\left(0.5 \rho D U_{\infty}^{2}\right)$ and $C_{L}=F_{y} /\left(0.5 \rho D U_{\infty}^{2}\right)$, where $F_{x}$ and $F_{y}$ are the total force in the in-line and transverse directions, respectively. The Strouhal number is defined as, $f_{S t}=f D / U_{\infty}$, where $f$ is the dominant frequency calculated from the time history of the lift coefficient.

The present model is further validated against flow around a stationary cylinder with $R e$ ranging from 75 to 250 . Figure 2 compares the predicted $f_{S t}$ with the published data such as $f_{S t}=0.2731-1.1129 / \sqrt{R e}+0.4821 / R e$ (Williamson \& Brown 1998) and the numerical results by Leontini et al. (2013) and Jiang et al. (2016). The difference between the present results and those published is within $1 \%$ in most of the cases investigated.

Validations are also carried out for an in-line oscillatory cylinder placed in a steady current at $R e=$ 175. Figure 3 shows the variations of $C_{L}$, and the vortex shedding frequency $\left(f_{s}=f D / U_{\infty}\right)$ with the oscillation amplitude $(A / D)$ at an $f_{d}$ that is equal to the Strouhal number of an otherwise stationary cylinder $\left(f_{d} / f_{S t}=1\right)$. Figure 4 maps the locations of the onset of primary lock-on in the $\left(f_{d} / f_{S t}, A / D\right)$ plane, along with some published results. In general, the agreement is satisfactory. The above validations suggest that the model and the computational mesh are adequate for the purpose of the study.

(a)

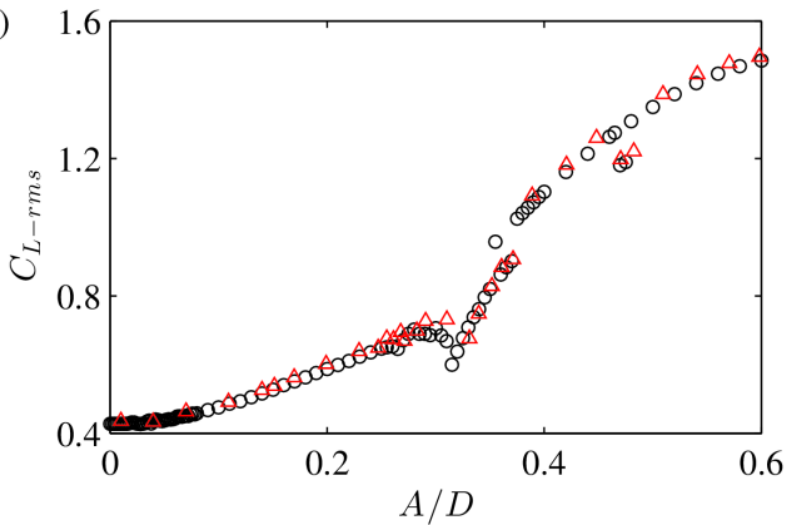

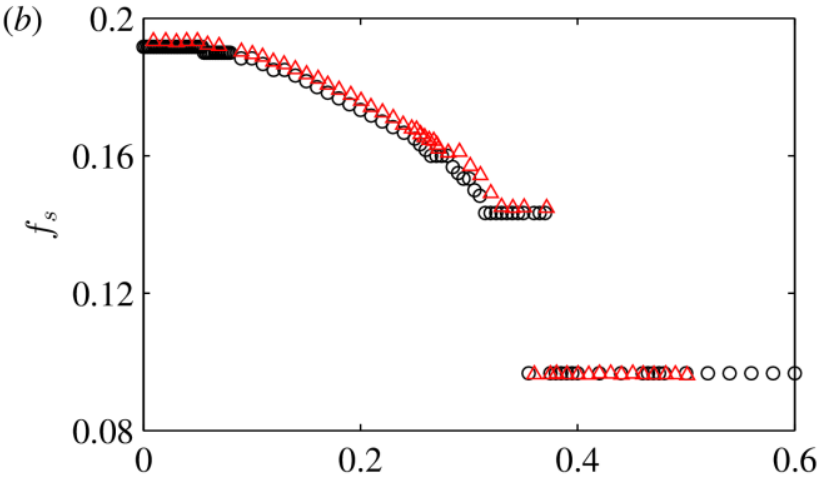

$A / D$

Figure 3 Comparison on the (a) rms lift coefficient $C_{L-\text { rms }}$ and (b) the vortex shedding frequency $f_{s}$ at $R e=175$ and $f_{d} / f_{S t}=$ 1. $\Delta$, Leontini et al. (2013); o, present study.

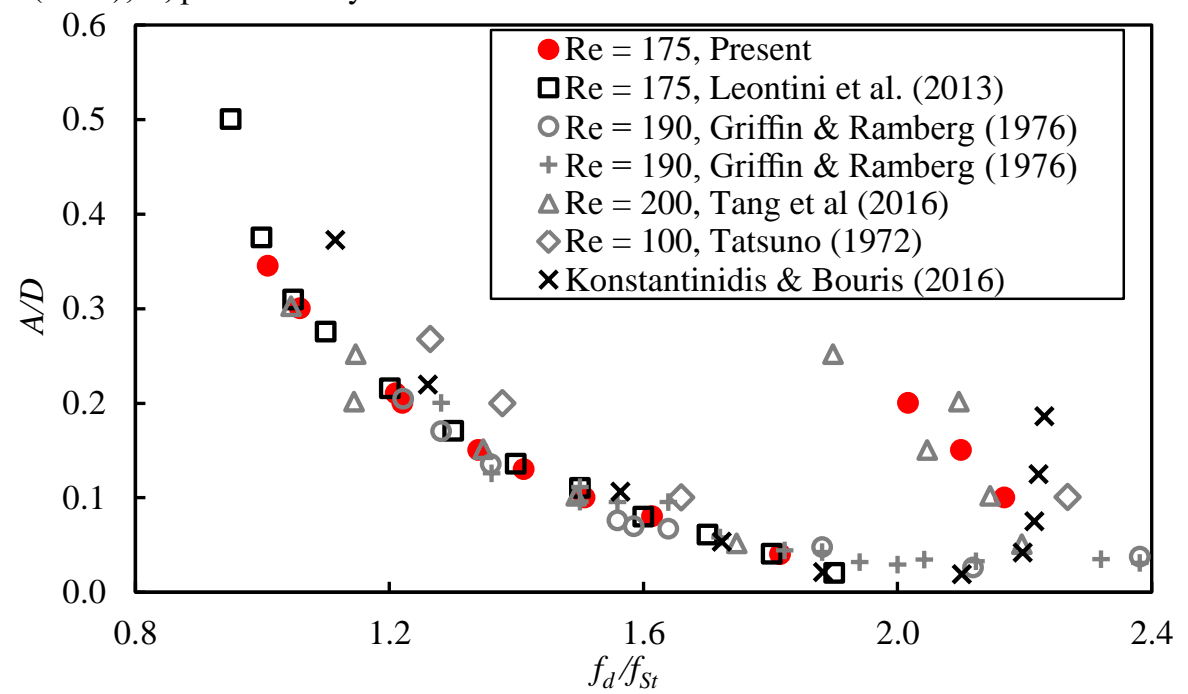

Figure 4 Comparison on the boundary of the primary synchronisation regime in the $\left(f_{d} / f_{S t}, A / D\right)$-plane map. Only available results for $R e \leq 200$ have been listed (Leontini et al. 2013; Griffin \& Ramberg 1976; Konstantinidis \& Bouris 2016; Tang et al. 2016) and data of Tatsuno (1972) were extracted from Barbi et al. (1986). 


\section{Numerical results}

\subsection{Identification of synchronisation}
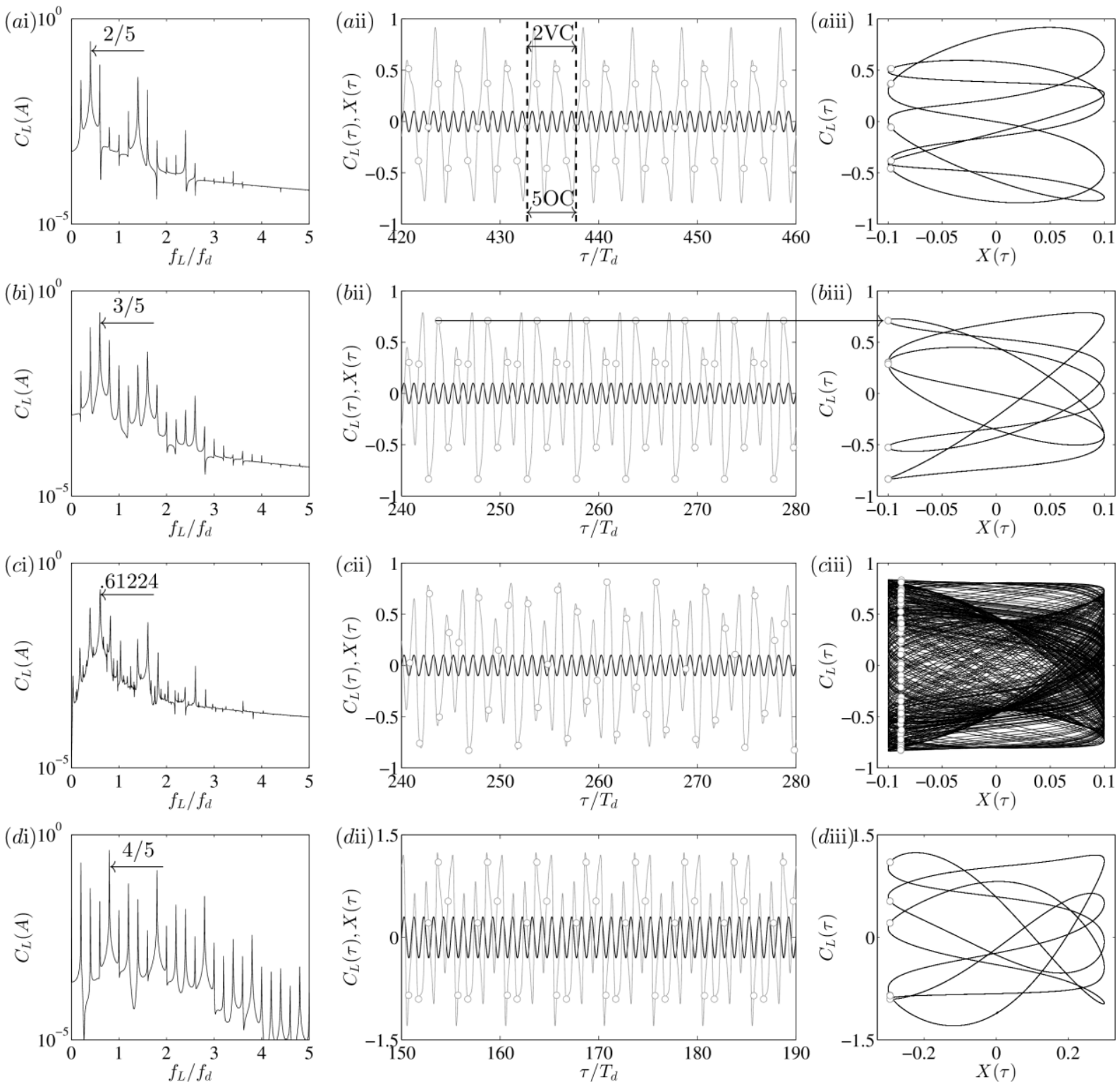

Figure 5 The synchronisations identification of an in-line oscillation circular cylinder in steady flow. From top to bottom, respectively, are the $p / q$ mode of $(a), 2 / 5$ at $\left(f_{d} / f_{S t}, A / D\right)=(2.45,0.1) ;(b), 3 / 5$ mode at $(1.48,0.1) ;(c)$, the nonsynchronisation or quasi-periodic mode at $(1.47,0.1)$; and $(d), 4 / 5$ mode, i.e. the $P_{5}$ mode, at $(1.00,0.3)$. From left to right columns are the (i) spectrum of $C_{L}$; (ii), transient trace of $C_{L}(\tau)$ (thin grey line) with cylinder displacement $X(\tau)$ (thick black line); and (iii), Lissajous phase diagram of $X(\tau)$ versus $C_{L}(\tau)$ with the Poincaré map (o) sampled at the driving frequency $\left(f_{d}\right)$ in 40 cycles of oscillation. The strongest peak in (i) is given (which coincides with vortex shedding frequency, $f_{s}$, and when synchronisation does occur this value is a fraction composed by natural numbers $\left(f_{s} / f_{d}=2 / 5,3 / 5\right.$ and 4/5). Meanwhile, the $X(\tau)$ and $C_{L}(\tau)$ phase diagram is closed, which has been indicated by the collapse of the Poincaré map, such as illustrated by the arrow running across (bii) to (biii). As illustrated in (aii), the mode ratio $p / q$ physically indicates $p$ vortex-shedding cycle (VC) of in $q$ cylinder-oscillation cycle (OC). Only part of the time histories in generating (iii) is shown in (ii) for clarity.

Firstly, the method used for identifying synchronisation is introduced. Apart from visual observations of flow fields, the time history of the lift coefficient has been employed for quantifying wake dynamics (Barbi et al. 1986; Al-Mdallal et al. 2007; Cetiner \& Rockwell 2001), partly because it is free from inertial forces acting along the direction of oscillation (Konstantinidis \& Bouris 2016). The identification of synchronization is usually achieved through examining the power spectrum 
obtained by using the Fast Fourier transform (FFT), the Lissajous phase diagram and the Poincare map. The synchronisation is characterised by the periodicity of $C_{L}$ and thus closed loops of the Lissajous phase diagram. Since forces are not always readily available in laboratory tests, velocity signals in the near wake are usually employed to identify the synchronisation mode (Ongoren $\&$ Rockwell 1988a). The time history and power spectrum of $C_{L}$, and the Lissajous phase diagram $\left(C_{L}\right.$ versus $X$ ) are used to identify synchronisation in the present study.

Figure 5 shows a few typical synchronisation and non-synchronisation cases based on $C_{L}(\tau)$ and $X(\tau)$, to explain how the modes of synchronisation are identified. Here, the frequency domain of the lift coefficient, $f_{L}$, in Figure 5(i) is normalized by the driving frequency, $f_{d}$, and the time $\tau$ by the driving period (Figure 5ii), $T_{d}=1 / f_{d}$. The Lissajous phase diagrams (Figure 5iii) for synchronisation cases at $\left(f_{d} / f_{S t}, A / D\right)=(2.45,0.1),(1.48,0.1)$ and $(1.00,0.3)$ are characterized by time-independent enclosed and regular loops. These cases represent three distinct synchronisation modes with $q=5$, which are further characterised by rational-ratio peaks $(p / q)$ in (i). On the other hand, the loops for an aperiodic case at $\left(f_{d} / f_{S t}, A / D\right)=(1.47,0.1)$ do not repeat themselves from cycle to cycle, where the phase trace lines will eventually fill up the whole phase space, given enough time (Ott 2002). The strongest peak for quasi-periodic or chaotic cases does not coincide with an identifiable rational ratio of $p / q$, such as that shown in Figure 5 (c).

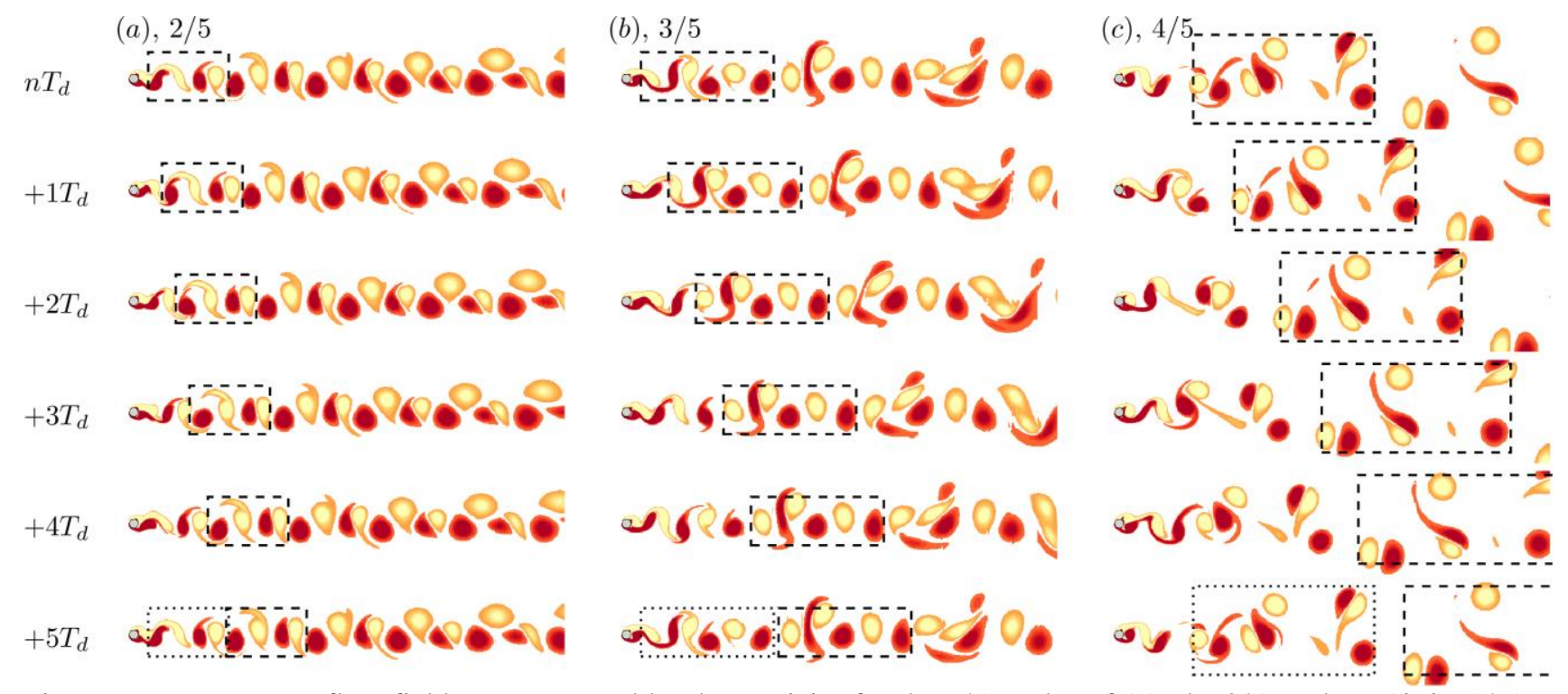

Figure 6 Instantaneous flow field as represented by the vorticity for the $p / 5$ modes of $(a)$, the $2 / 5$ mode at $\left(f_{d} / f_{S t}, A / D\right)=$ $(2.45,0.1) ;(b)$, the $3 / 5$ mode at $(1.48,0.1)$; and $(c)$, the $4 / 5$ mode at $(1.00,0.3)$, as shown in Figure 5. Dimensionless vorticity contours of $\omega_{z}$ are given from -0.5 (bright colours) to +0.5 (dark colours) with cut-off level \pm 0.1 . The dash box traces the movement of $p$ pairs of vortex in 5 cycles of oscillation.

It is understood that the maximum peak in Figure 5(i) corresponds to the vortex shedding frequency, $f_{s}$. For synchronisation cases, the physical meaning of the ratio $p / q=f_{s} / f_{d}$ is that there are $p$ cycles of vortex shedding in $q$ cycles of cylinder oscillation. Therefore, $p$ and $q$ can be identified by counting the repeated pattern of $C_{L}(\tau)$ and $X(\tau)$ in (ii). In this example, 2, 3 and 4 cycles of lift oscillations are observed in 5 cycles of cylinder oscillations, which are now referred to as the $2 / 5$ mode, $3 / 5$ mode and 4/5 mode of synchronisation, respectively. It is noted that the $1 / 5$ mode is out of the range of the present work and in fact it shows up at around $f_{d}=4.5 f_{S t}$ for a cylinder oscillating transversely to the flow as reported by Ongoren \& Rockwell (1988a). It is also worth pointing out that the $5 / 5$ mode is normally identical to the $1 / 1$ mode (identified elsewhere), which also serves as an example that an incompletely reduced fraction, such as 6/8 (identical to $3 / 4$ ), is not a mode commonly identified in the present work. 
The typical flow fields for these $p / 5$ modes are shown in Figure 6 by vorticity contours of $\omega_{z}$, calculated using $\nabla \times \boldsymbol{U}$. The inset boxes highlight the shed and convection of 2, 3, and $4(p)$ pairs of vortices in $5(q)$ cycles of oscillation, respectively. Similar vortex shedding processes to those shown in Figure 5 are also observed for other $p / q$ modes, which will not be shown in details for ease of the presentation.

\subsection{Modes of synchronisation}

Numerical simulations have been carried out for $f_{d} / f_{S t} \in[0.8,4]$ and $A / D \in[0,0.5]$ at a fixed $\operatorname{Re}=$ 175 (based on $U_{\infty}$ and $D$ ) with fine increments of $A / D$ and $f_{d} / f_{S t}$. The simulations lasted for approximately $\tau=U_{\infty} t / D \geq 1000$ to ensure that an equilibrium state of the flow has been established. All identified cases with synchronisation are shown in Figure 7 in the $\left(f_{d} / f_{S t}, A / D\right)$-plane. For simplicity, different symbols are only used for synchronisation modes with $q$ (and $p$ ) $\leq 10$, and a diverse range of long-period modes $(p>10)$ of synchronisation are classified into other modes $(\mathrm{OM})$. Although a much larger set of simulations than those shown in Figure 7 were carried out, the flows deemed non-synchronisation are excluded for clarity. The non-synchronisation flows are referred to as quasi-periodic or chaotic flows hereafter. Admittedly, there are possibly long-period synchronisation modes in these excluded cases, which are not easily identifiable from the Lissajous phase diagram.

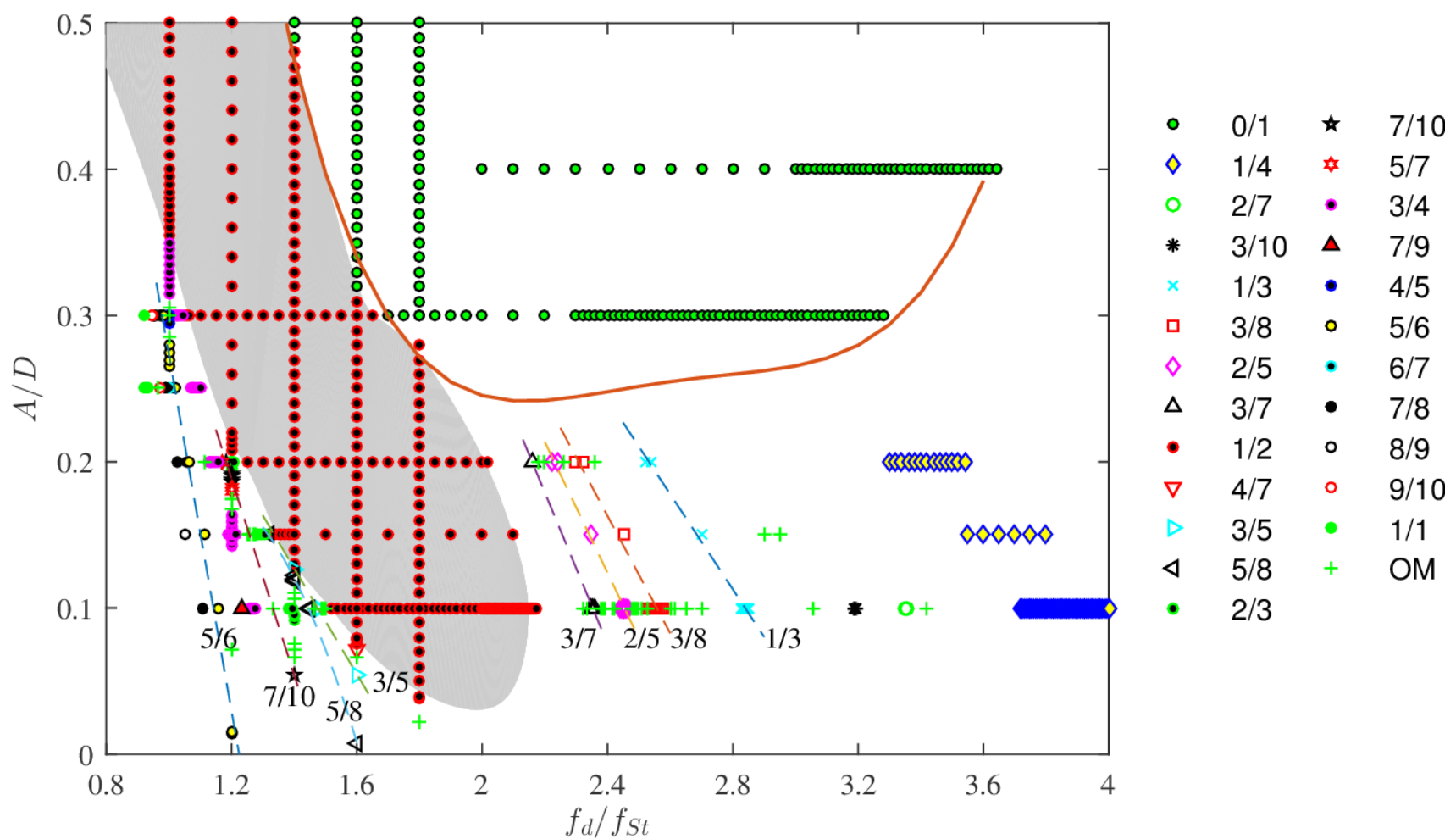

Figure 7 Locations of the synchronisation modes of an in-line oscillatory cylinder in the steady flow in the $\left(f_{d} / f_{S t}, A / D\right)$ plane. Modes of synchronisation composed of natural numbers equal or smaller than 10 have been listed in the legend with the increase value of mode ratio, while all other modes $(\mathrm{OM})$ are represented by + . All cases deemed quasi-periodic or chaotic have been removed for clarity. A single line of approximation has been drawn for the selected modes of synchronisation $(5 / 6,7 / 10$ and others), although it is noteworthy that each mode dominates a band in the controlling space, narrow (such as modes $3 / 7$ and 5/8) or wide (modes $1 / 2$ and 1/4). The shaded area indicates the location of $1 / 2$ mode, while the solid line is a curved-fitted line for the boundary of mode $0 / 1$.

At approximately $A / D<0.3$, Figure 7 shows the existence of a series of modes of synchronisation. It is seen whenever $f_{d}$ is in the vicinity of $N$ times $f_{S t}, f_{d} \sim N f_{S t}$ (e.g. 2, 3 and 4), the flow (vortex shedding) locks-on to $(1 / N) f_{d}$, or more generally, whenever $f_{d}$ is in the vicinity of a rational ratio of 
the natural frequency, $f_{d} \sim(q / p) f_{S t}$, then the vortex shedding locks-on to $f_{s}=(p / q) f_{d}$, where $N, p$ and $q$ are integers. From the perspective of nonlinear dynamical systems, Olinger \& Sreenivasan (1988) referred these bands of synchronisation as Arnold's tongues, interleaved in the quasi-periodic cases. Except the two dominant modes (1/2 and 1/4), the bands of synchronisation (composed by cases of the same mode at different controlling parameters, as indicated by the lines of approximation at selected modes) are mostly very narrow. The cylinder oscillation serves as a disturbance to the fundamental vortex shedding process induced by the steady flow. A general trend is that the $f_{d}$ required for a certain mode reduces as the level of disturbance $(A / D)$ increases, in agreement with the $P_{N}$ synchronisation map by Leontini et al. (2013).

As observed in Figure $7, f_{s} / f_{d}$ locks-on to almost all possible completely reduced rational ratios composed of natural numbers smaller than 10 and some more above, including the primary lock-on mode (Bishop \& Hassan 1964; Tanida et al. 1973) and the $P_{N}$ mode (Leontini et al. 2013). It also appears that the mode of synchronisation favors small natural numbers $(p$ and $q$ ), and the integer combination of $1 / 2$ dominates in the parameter space for $A / D<0.3$. This, understandably, is the one of the reasons that a wealth of research has been devoted to the $1 / 2$ mode. The next dominant mode is $1 / 4$ from the map, followed by modes such as 3/4 and 1/3, which are the earliest reported modes of synchronisation as documented by Ongoren \& Rockwell (1988a).

It is found that fine increments of $A / D$ and $f_{d} / f_{S t}$ are required in systematically capturing synchronisation modes composed of relatively large natural numbers of $p$ and $q$, such as 3/5, 3/8, 3/7 and $7 / 10$. Those modes were seldom reported in the published work as cited earlier. A fascinating feature of the flow in relatively large-integer modes is that the periodicity is maintained for a long period of seemingly disordered oscillations. Some example cases will be given later on to show the complexity of the flow field.

It is estimated that there are approximately $35 \%$ of the total investigated cases belong to the synchronization state for $A / D \leq 0.3$. Thus at low amplitude the synchronised phenomenon is not a common feature, but the resonance serves as the limiting case in a series of quasi-periodic responses, as discussed by Lotfy \& Rockwell (1993), which therefore provides an insightful perspective to the wake flow.

\subsection{1. $\quad$ Mode $f_{s} / f_{d}=0 / 1$}

The parameter space with $A / D>0.3$ and $f_{d} / f_{S t}>1.2$ as shown in Figure 7 is occupied by symmetric flows with regards to the oscillation direction, especially close to the cylinder. The existence of such a region is due to the large disturbance induced by the oscillation that significantly affects the inherent vortex shedding. This flow feature is classified as the $0 / 1$ mode of synchronisation to represent the strong suppression on natural vortex shedding. If $C_{L-r m s}$ is smaller than $10^{-3}$, the natural vortex shedding is deemed to be completely suppressed $\left(f_{s}=0\right)$, leading to another large area in the $\left(f_{d} / f_{S t}, A / D\right)$-plane that is labelled as $f_{s} / f_{d}=0 / 1$. The wake characteristics associated with symmetric flows are shown in Figure 8 at $A / D=0.4$ with a range of $f_{d}$. In the experimental study by Konstantinidis \& Balabani (2007), symmetric flows were promoted for $f_{d}$ around $3 f_{S t} \sim 4 f_{S t}$ at $R e=$ 1200 , where the symmetry was only sustained in the neighborhood of the oscillatory circular cylinder, giving rise to asymmetric arrangements of vortex structures further downstream. In the present simulations, asymmetric far wake is also found on both ends of $f_{d}$ for the band of mode $0 / 1$ but the wake can also be perfectly symmetric throughout the simulation domain for intermittent $f_{d}$. The shedding of vortices is mainly induced by the cylinder oscillation rather than the steady flow at mode $0 / 1$, where the shedding of vortices due to cylinder oscillation weakens the shear layers induced by the steady flow, resulting in the disappearance of the conventional Kármán vortex street. 
A similar steady solution from the NS equations at large $\operatorname{Re}(>50)$ was also found in the wake of a rotational cylinder (Rao et al. 2013). As $f_{d}$ further increases beyond $f_{d} / f_{S t}=3.4$, the conventional Kármán vortex street returns mainly because vortex shedding induced by cylinder oscillations becomes desynchronised with the driving frequency.
$(a), f_{d} / f_{S t}=1.6$
(b), 1.8
$(c), 2.0$

(d), 2.9

\section{का}

$(g), 3.5$ (e), 3.1

(f), 3.3

$(h), 3.7$

(i), 3.9

\section{0.

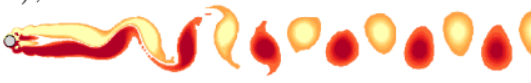 \\ 000000000}

Figure 8 Flows development with increasing driving frequency inside and outside of the deemed $f_{s} / f_{d}=0 / 1$ regime at $A / D$ $=0.4$.
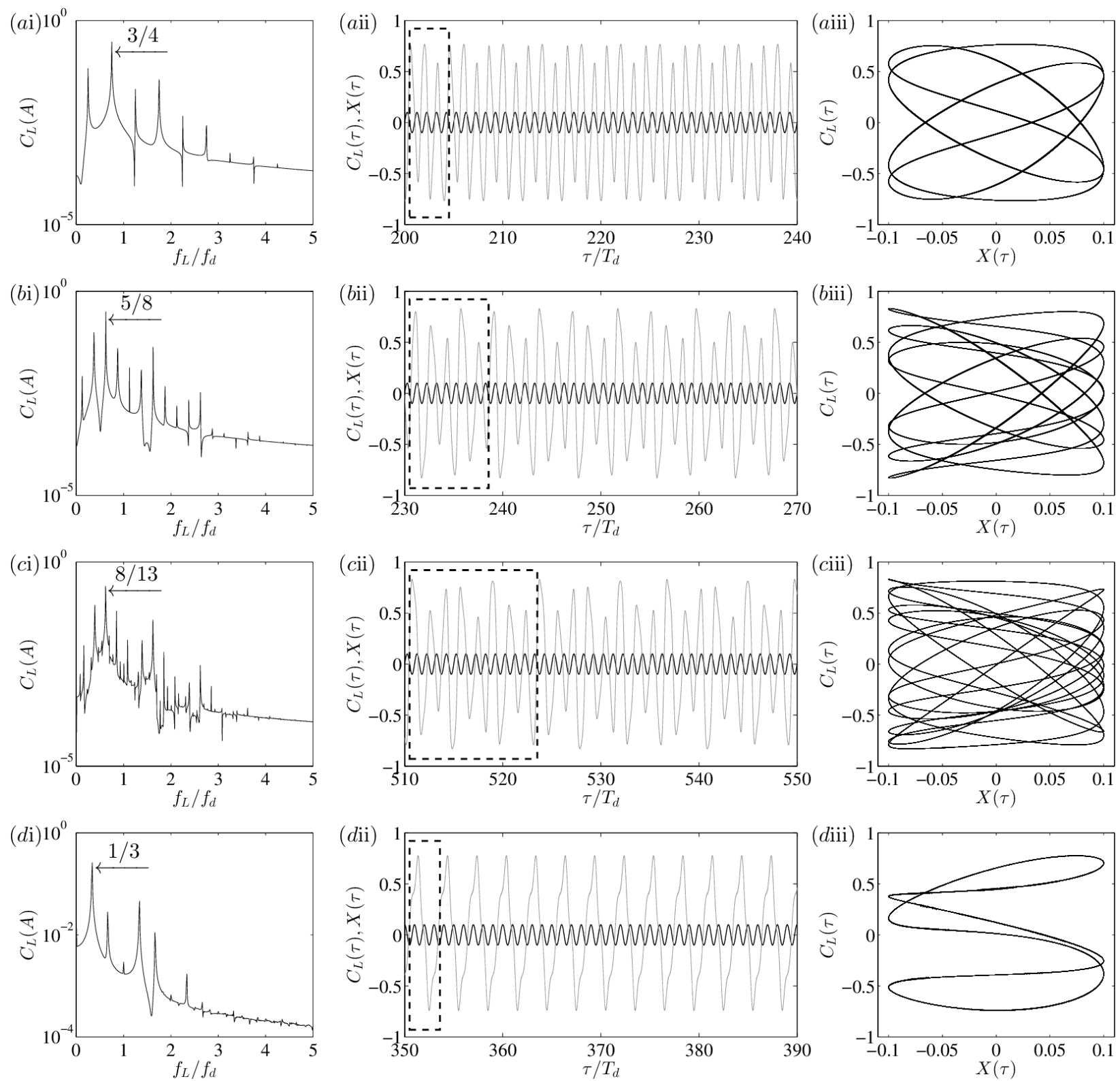
Figure 9 Selected modes of synchronisation at a fixed amplitude of oscillation, $A / D=0.1$, but different driving frequencies, from top to bottom at $(a), f_{d} / f_{S t}=1.260 ;(b), 1.445 ;(c), 1.460$ and $(d), 2.825$, respectively. The dashed box encloses $X(\tau)$ and $C_{L}(\tau)$ in a synchronisation period. Refer to Figure 5 for more captions.

\subsection{2. $\quad$ Selected modes at $A / D=0.1$}

Flow synchronisations with large $p$ and $q$ values, such as the 8/13 mode, are illustrated in Figure 9, along with the flow fields in Figure 10. The modes are chosen to highlight the subharmonic modes of the present interest and to compare them with those reported elsewhere. The periodicity of the $8 / 13$ mode is quite subtle compared with the modes with small $p$ and $q$ values, such as the $1 / 3$ mode. The time history of $C_{L}$, as observed in the inserted box in Figure 9(cii), seems to lose its periodicity, in both amplitudes and the zero-crossing intervals until after 13 exciting cycles, where $C_{L}$ has experienced 8 cycles of oscillation and starts to repeat itself in exactly the same manner thereafter. The detection of synchronisations with large $p$ and $q$ values through $C_{L}(\tau)$ or flow fields as shown in Figure $10(c)$ is not as straightforward as from the Lissajous phase diagram.

The mode of 5/8 shown in Figure 9(b) is interesting in the sense of the distinct spectrum peaks in the frequency domain, where approximately 20 equally spaced peaks appear among $f_{L} / f_{d} \in[0,5]$. The distribution peaks among $[0,1]$ are self-similar to those in $[1,2],[2,3]$, and so on. This regularity demonstrates the perfect periodicity of the flow, which once again is vague in the flow field shown in Figure 10(b). The similarity in the distribution of spectrum peaks as a function of $f_{L} / f_{d}$ is also evident in other cases $(a, c$ and $d)$ and the spectrum content will be further discussed in section 4.4 .
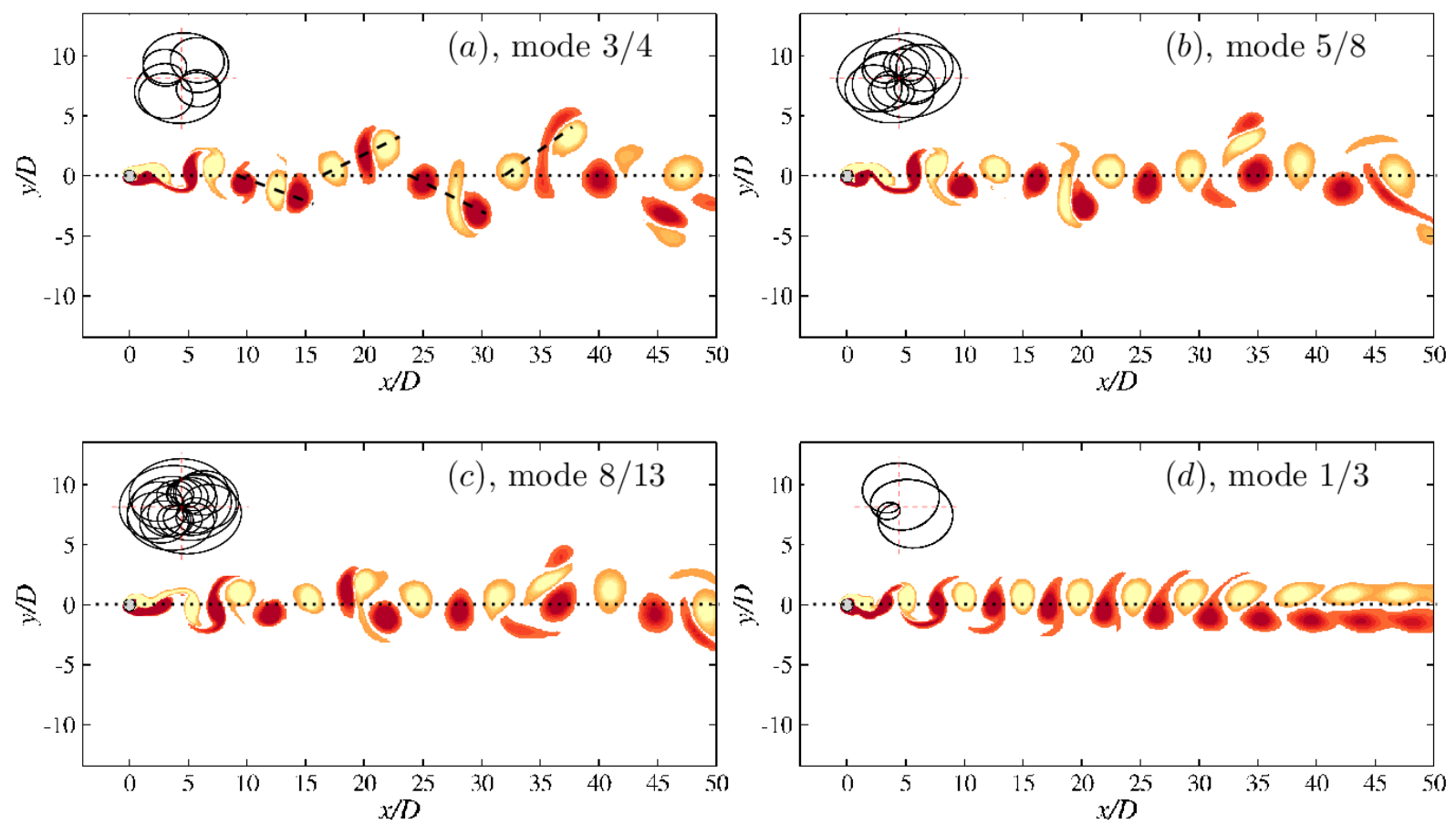

Figure 10 Instantaneous vorticity field $\left(\omega_{z}\right)$ for the same modes of synchronisation as given in Figure 9 . The inset diagrams are the phase portrait of the lift coefficient with two crossed-dashed lines indicating the origin of the coordinates.

Identifying the lock-on modes from wake features works the best at modes with small $p$ and $q$ integers. Figure 10(a) demonstrates this with a 3/4 mode where three pairs of vortices (shed in four cycles of oscillation) are rearranged into two groups (indicated by inclined-dashed lines) in the wake. This simplicity makes it easily detectable and thus similar flows have been investigated intensively. 
For example, a similar flow has been documented as the $\mathrm{P}+\mathrm{S}$ mode by Williamson \& Roshko (1988), the A-III mode by Ongoren \& Rockwell (1988b), vortex triplets by Detemple-Laake \& Eckelmann (1989), as well as the $P_{4}$ mode by Leontini et al. (2013). This is likely the reason that little has been reported on the wake structures of the high-order subharmonic modes. A review on the wake flow classifications can be found in Konstantinidis \& Bouris (2016).

The insets in Figure 10 show the phase portraits of $C_{L}$, which have been calculated according to Baek \& Sung (2000) as

$$
a(\tau)=C_{L}(\tau) \sin (\omega \tau), \quad b(\tau)=C_{L}(\tau) \cos (\omega \tau) .
$$

The phase portrait separates two orthogonal components from $C_{L}(\tau)$ with respect to the carrier frequency. The definition suggests that from any point on the phase portrait, the distance to the coordinate origin $(0,0)$ represents the amplitude of $C_{L}(\tau)$, with an instantaneous phase angle of $\theta=\arctan (a(\tau) / b(\tau))$. When using $f_{d}$ as the carrier frequency, $\omega=2 \pi f_{d}$, the phase portrait yields a limited number of loops for the synchronised flow, which is the case for all modes shown in Figure 10. The number of loops appearing in the phase portrait is dependent on $p$ and $q$ in the mode ratio and the phase portrait becomes increasingly complicated as $p$ and $q$ become large.
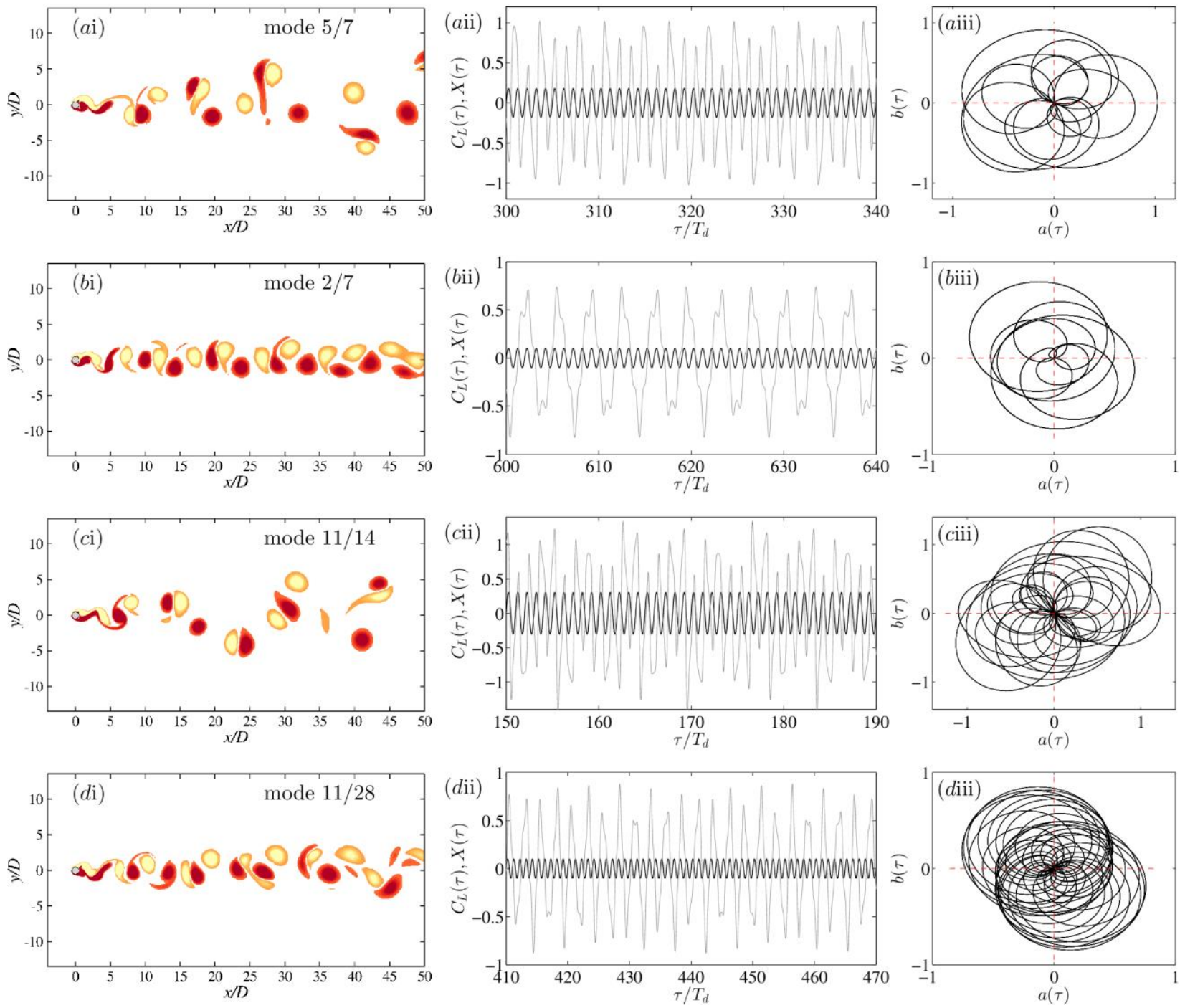

Figure 11 Synchronisation modes of $f_{s} / f_{d}=p / 7$ and $11 / q$. From top to bottom are $(a),\left(f_{d} / f_{S t}, A / D\right)=(1.2,0.180) ;(b)$, $(3.355,0.1) ;(c),(1.0,0.305)$ and $(d),(2.480,0.1)$, respectively. Refer to Figure 9 and Figure 10 for more captions. 


\subsection{3. $\quad$ Selected modes vs prime numbers}

Many synchronisation mode ratios involve a prime number, which is necessary to form completely reduced rational ratios. The flow features for such modes are illustrated in Figure 11 at two prime numbers that constitute the modes of $p / 7$ and $11 / q$. A common feature of these modes is that the phase portrait is simple when the mode ratio is composed of small integers, but complex with large integers. It is found that modes with large integers are sensitive to the controlling parameters. The synchronisation feature observed in mode $11 / 28$ in Figure $11(d)$ changes with a slight change in $f_{d}$, $A / D$ or Re. It is noted that Konstantinidis \& Bouris (2016) have recently demonstrated that even the strongest lock-on mode $1 / 2$ is also dependent on the initial conditions, especially near its regime boundaries.

\subsubsection{Beating feature}

A number of quasi-periodic cases is observed where the flow shows an asymptotic state to synchronisation. The quasi-periodicity in the flow is thought to be due to the interaction of at least two incommensurable frequencies including $f_{s}$ and $f_{d}$. Figure 12 gives such an example, in which $C_{L}(\tau)$ apparently displays a beating phenomenon. The chosen case at $\left(f_{d} / f_{S t}, A / D\right)=(2.220,0.1)$ is close to the boundary of the $1 / 2$ mode (see Figure 7), where the frequency content $f_{L} / f_{d}$ around $1 / 2$ in the FFT spectra is affected by two frequencies which are comparable in strength. There are plenty of similar quasi-periodic states found in the simulations. Baek \& Sung (2000) demonstrated that, in the quasi-periodic wake flow, the driving, natural and modified vortex shedding frequencies are all significant and compete with each other.
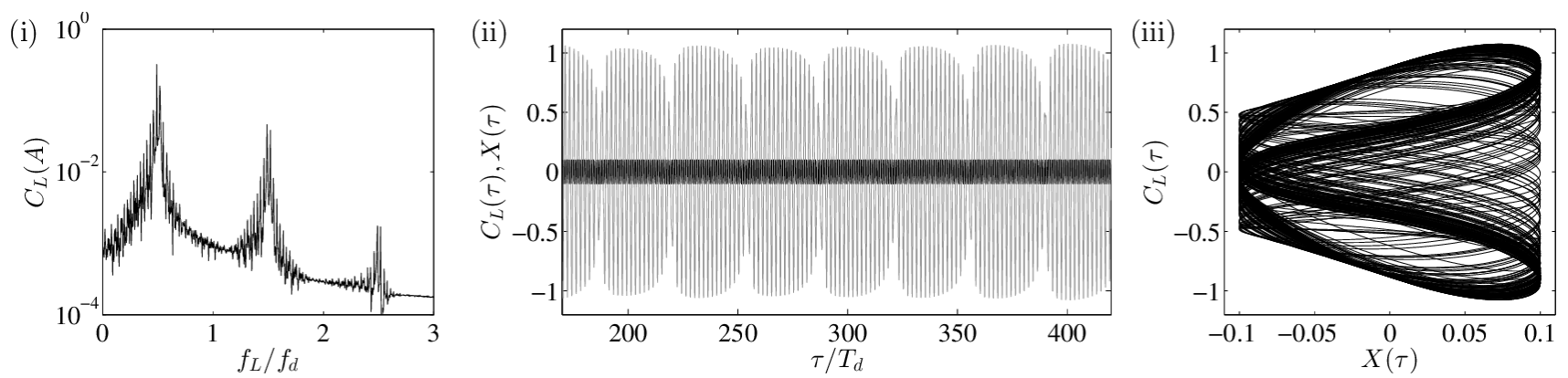

Figure 12 A beating feature displayed by the lift coefficient at $\left(f_{d} / f_{S t}, A / D\right)=(2.220,0.1)$. Refer to Figure 5 for more captions.

\section{Discussion}

\subsection{Synchronisation modes available}

Available synchronisation modes are inferred based on the Farey sequence in number theory. The numerical results presented have shown that $f_{s} l f_{d}$ is always a rational ratio between 0 and 1 when the subharmonic synchronisation occurs. The available rational ratios among $[0,1]$ that are composed of natural numbers smaller than 10 are shown in Table 2, which is known as the Farey sequence. The first column of the table lists the order of the sequence and the rest of columns list the rational ratios. A high order row includes rational ratios from all lower orders. The Farey sequence may provide useful guidance for identifying potential synchronisation modes.

It is found that almost all of the rational ratios enclosed in the dashed box in Table 2 correspond to a synchronisation mode (except $4 / 9$ and 5/9, which will be explained later on). It is inferred that the rational ratios in the Farey sequence form all available modes of synchronisation for an oscillatory cylinder in a steady current. It is seen from Table 2 that the gap between two neighboring numbers in 
each row decreases with increased order of the sequence $(q)$. This corresponds to the requirement of the fine-frequency increment to capture high-order synchronisation modes.

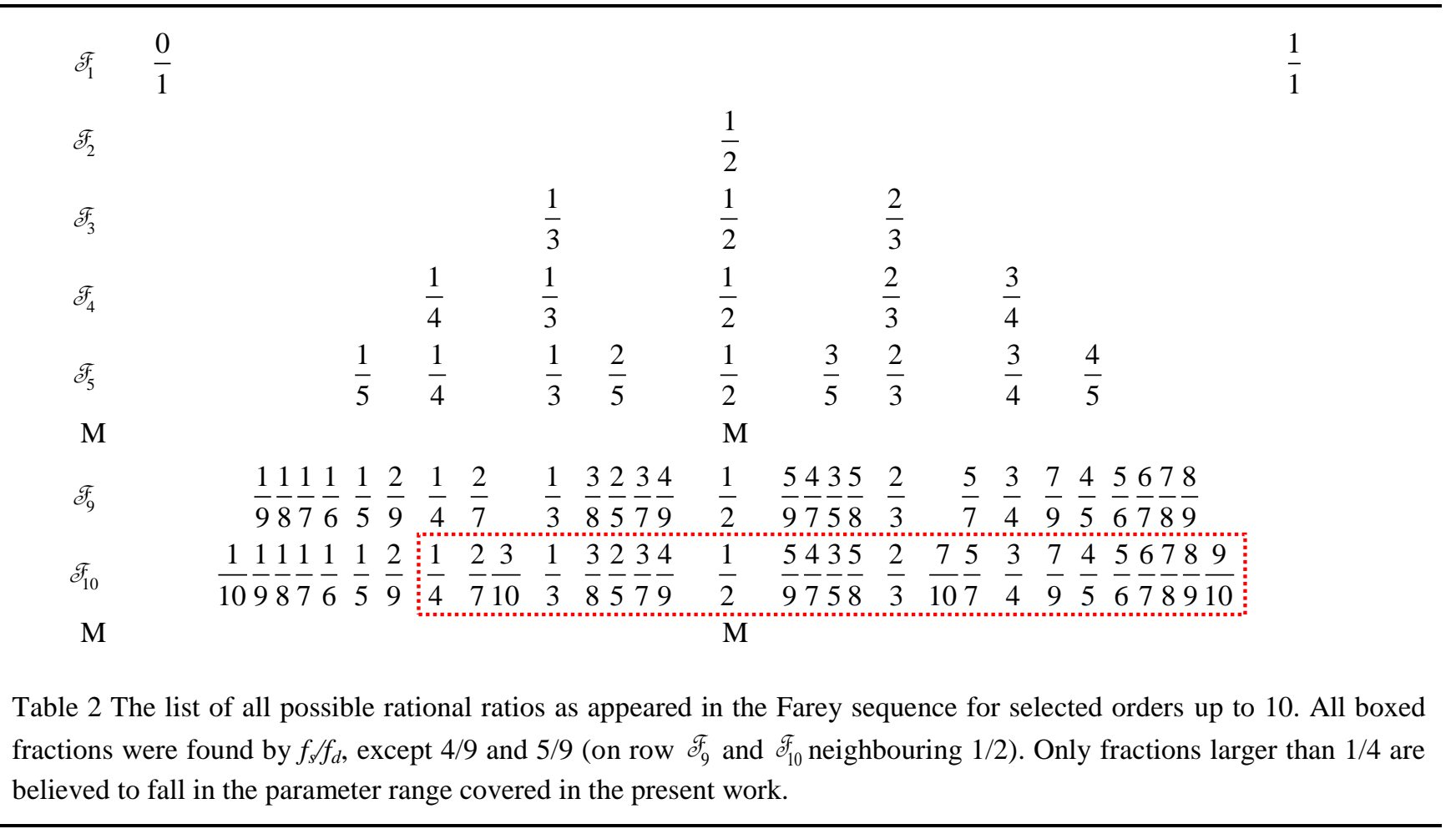

Lower order ratios appear firstly in the sequence and they generally occupy large area in the table, which is consistent with modes of synchronisation in the $\left(f_{d} / f_{S t}, A / D\right)$-plane map shown in Figure 7. Higher order ratios tend to emerge in a confined area, such as in the bottom corner area on the right of the table. Specifically, there are only three available modes of synchronisation from 4/9 $\sim 5 / 9$ $(0.44 \sim 0.56)$ (see order 10 in Table 2), while there are six over a similar size of the interval from $4 / 5$ $\sim 9 / 10(0.8 \sim 0.9)$. This distribution of available rational ratios concurs with the mode selection of the flow. A general observation from the numerical results is that the wake tends to adjust itself to a frequency that is commensurate with the oscillation. In such a way, fluid flow has fewer available states in between $4 / 9 \sim 5 / 9(0.44 \sim 0.56)$ than that between $4 / 5 \sim 9 / 10$, where in the former range the flow presents the primary lock-on feature, and in the latter range the flow feature may change with a slight change in the controlling parameters. The reason that synchronisation modes of 4/9 and 5/9 were not found in this study is likely due to their closeness to the dominant mode $1 / 2$. Since the inline force typically fluctuates at twice the frequency of the transverse force, so that driving at $2 f_{S t}$ leads to the primary synchronization between the vortex shedding and the oscillation (Bishop \& Hassan 1964; Leontini et al. 2011). The primary mode is so strong that it overlaps with other modes. As the oscillation amplitude is increased in Figure 7, the frequency range covered by mode $1 / 2$ also gradually widens till $A / D \approx 0.25$ and thus more missing synchronisation modes are expected within the area occupied by mode $1 / 2$.

There are potentially an infinite number of available mode ratios if we consider the orders of the Farey sequence greater than 10 in Table 2. An interesting question arising from this is whether can we predict the locations of occurrence of the available modes (Table 2$)$ in the $\left(f_{d} / f_{S t}, A / D\right)$-plane. The answer can be found out from the results shown in Figure 7 and Table 2, which suggest that the occurrence of a particular synchronisation mode $p / q$ is dependent on both $f_{d} / f_{S t}$ and $A / D$. First of all, synchronisation mode $p / q$ would normally occur in the vicinity of $1 /\left(f_{d} / f_{S t}\right)$ that is approximately equal to $\mathrm{p} / \mathrm{q}$. For examples, synchronisation modes $1 / 2$ and $1 / 4$ occur in the vicinity of $f_{d} / f_{S t}=2 \& 4$ 
respectively. Secondly, an increase in $A / D$ encourages the synchronisation to occur at a smaller value of $f_{d} / f_{S t}$ than the corresponding $f_{d} / f_{S t}$ at which the synchronisation is supposed to occur. That is the reason why the synchronisation trend bands (indicated by dashed lines in Figure 7) lean towards the left as $A / D$ increases. The above observations provide a general guidance on the approximate locations of occurrence of available modes in the $\left(f_{d} / f_{S t}, A / D\right)$-plane.

\subsection{Wake characteristics and lift coefficient}

The wake characteristics are illustrated by examining the absolute mean lift coefficient, $\left|\bar{C}_{L}\right|$, as a function of $f_{d} / f_{S t}$ at $A / D=0.1$ and 0.2 in Figure 13. A zero-mean $C_{L}(\tau)$ suggests an equal distribution of vortices/energy besides the cylinder with respect to the oscillation direction. For synchronised flows with an even-denominator or quasi-periodic flows, the $\left|\bar{C}_{L}\right|$ is generally close to zero. This observation quantitatively demonstrates that a relatively evenly distributed wake is the preferred wake formation. The synchronised flow with an even-denominator mode generally maintains an adjusted spatial-temporal symmetry as,

$$
\omega_{z}(x, y, \tau)=-\omega_{z}\left(x,-y, \tau+T_{M} / 2\right) \text {, }
$$

where $T_{M}$ is the period of synchronisation. This is especially clear at low $A / D$ and at rational ratios composed of small integers. The symmetry induces a global wake oscillation at a frequency $\left(f_{g} / f_{d}=\right.$ $2 p / q$ ) that corresponds to the shedding frequency of two groups of $p$ vortices. The $3 / 4$ mode flow shown in Figure 10(a) is a typical of such flows.

On the other hand, it is observed that mode ratios of $2 / 3,3 / 5,3 / 7,2 / 5$ and $1 / 3$ present distinct peaks in $\left|\bar{C}_{L}\right|$. This is due to the uneven distributions of vortices on either side of the oscillation axis and mainly corresponds to a synchronised wake with an odd-denominator mode ratio, for instance in Figure $10(d)$ where more vortices are located above $y=0$. The flow maintains a spatial-periodic state over the whole synchronisation period as

$$
\omega_{z}(x, y, \tau)=\omega_{z}\left(x, y, \tau+T_{M}\right)
$$

The range of $f_{d} / f_{S t}$ over which the occurrence of the odd-denominator modes occurs is narrow, mostly including only a couple of cases.

By comparing Figure 7 and Figure 13, it is found that the flow favors synchronisation modes with an even-number denominator over those with an odd-number denominator. There are far more cases, for instance, with $3 / 4$ or $5 / 6$ mode ratios than that of $4 / 5$ in Figure 7 . It is interpreted as that both the background steady flow and the oscillatory perturbations affect the distribution of wake vortices, but the steady flow is the dominant mechanism at low $A / D$. Since Kármán vortex street has the spatial symmetry about the flow direction in equation (4-1), the overall flow favors the symmetric state for this reason. The uneven distribution of vortices to the direction of oscillation is believed to be a manifestation of the asymmetric vortex formation induced by the cylinder oscillation, including the flow field of mode 1/2 as given in Figure $13(b)$. In the case of oscillatory cylinders in still water, the shed vortices can move laterally to the axis of oscillation (Tatsuno \& Bearman 1990; Tong et al. 2015; Tong et al. 2017).

One point that is worth mentioning related to mode $2 / 3$ is that it is a special mode in terms of small integer numbers (only second to mode $1 / 2$ in this sense). The mode $2 / 3$ is less dominant than, for instance modes $3 / 4$ or $3 / 8$. In fact, attributing to the narrow synchronisation region, mode $2 / 3$ has rarely been reported previously. As discussed above, the odd denominator requires an inclined wake, 
but the steady flow dominates the wake formation. Instead, around the location of mode $2 / 3$, the flow is sensitive to the variation of $f_{d}$ and changes accordingly to either a quasi-periodic state (another way to equally distribute the rotational flow) or a nearby synchronisation state with an even denominator (such as the 3/4 mode). In one case, the flow even synchronises at an incompletely reduced mode ratio, 4/6, and this adjustment brings $\bar{C}_{L}$ close to zero, as indicated in Figure 13(b).
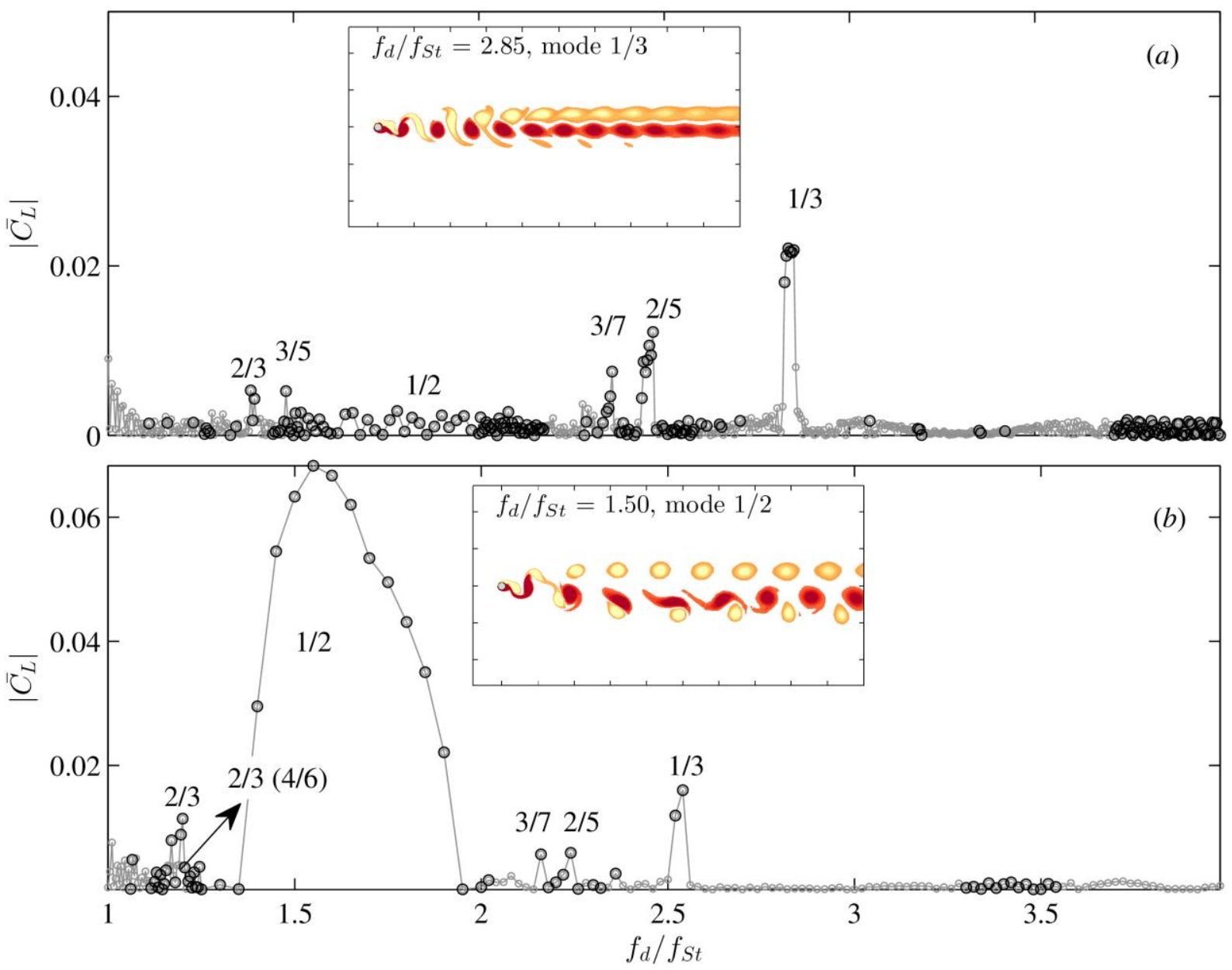

Figure 13 The absolute value of the mean lift coefficient at $(a) A / D=0.1$ and $(b), A / D=0.2$ as a function of driving frequency. Grey $\circ$ give all the simulated cases, while slightly larger black $\circ$ are the cases deemed synchronisation. The insets are two selected flow fields at the two amplitudes of oscillation.

\subsection{Frequency adjustment}

The influence of cylinder oscillations on $f_{s}$ is illustrated in Figure $14(a, b)$ at a constant value of $A / D=0.1$, with a frequency increment $\Delta\left(f_{d} / f_{S t}\right)$ as fine as 0.005 at certain locations. Comparing with natural vortex shedding, cylinder oscillations slow down the vortex shedding process at most $f_{d} / f_{S t}$, as shown in Figure $14(a)$. In the range of $f_{d} / f_{S t}=1 \sim 2$ the reduction of $f_{s} / f_{S t}$ is generally continuous if the lock-on cases are excluded, in agreement with Leontini et al. (2013). However, such a trend is lost for large $f_{d}$. Undoubtedly, $f_{s}$ shows a linear response once it is synchronised to the oscillation (in each of the modes highlighted by the dashed lines) and exceeds $f_{S t}$ at the end of modes $1 / 2$ and $1 / 4$. In total $f_{s}$ ranges approximately from $75 \%$ to $110 \%$ of $f_{S t}$, and the extreme values are found at the two ends of the 1/2 region, respectively. Another prominent feature in Figure 14 is the discontinuities in the diagram, which are mainly due to the regime jumps of the flow, between different synchronised modes, or between synchronised and desynchronised cases.

When normalized by $f_{d}$ as given in Figure $14(b), f_{s} / f_{d}$ shows a few plateaus (as indicated by the 
horizontal dashed lines), which were referred to as the 'Devil's-staircase' by Olinger \& Sreenivasan (1988) in the perspective of a low-dimensional dynamic system, to highlight the discontinuous state in the frequency adjustment. These plateaus are interleaved with the quasi-periodic cases. The synchronised mode ratio $f_{s} / f_{d}$ therefore experiences a consistent drop with increasing $f_{d} / f_{S t}$, but whenever $f_{s} / f_{d}$ drops to a rational ratio, this trend temporarily stops (linear response) until the flow is disturbed further by the increased exciting frequency (non-linear response).
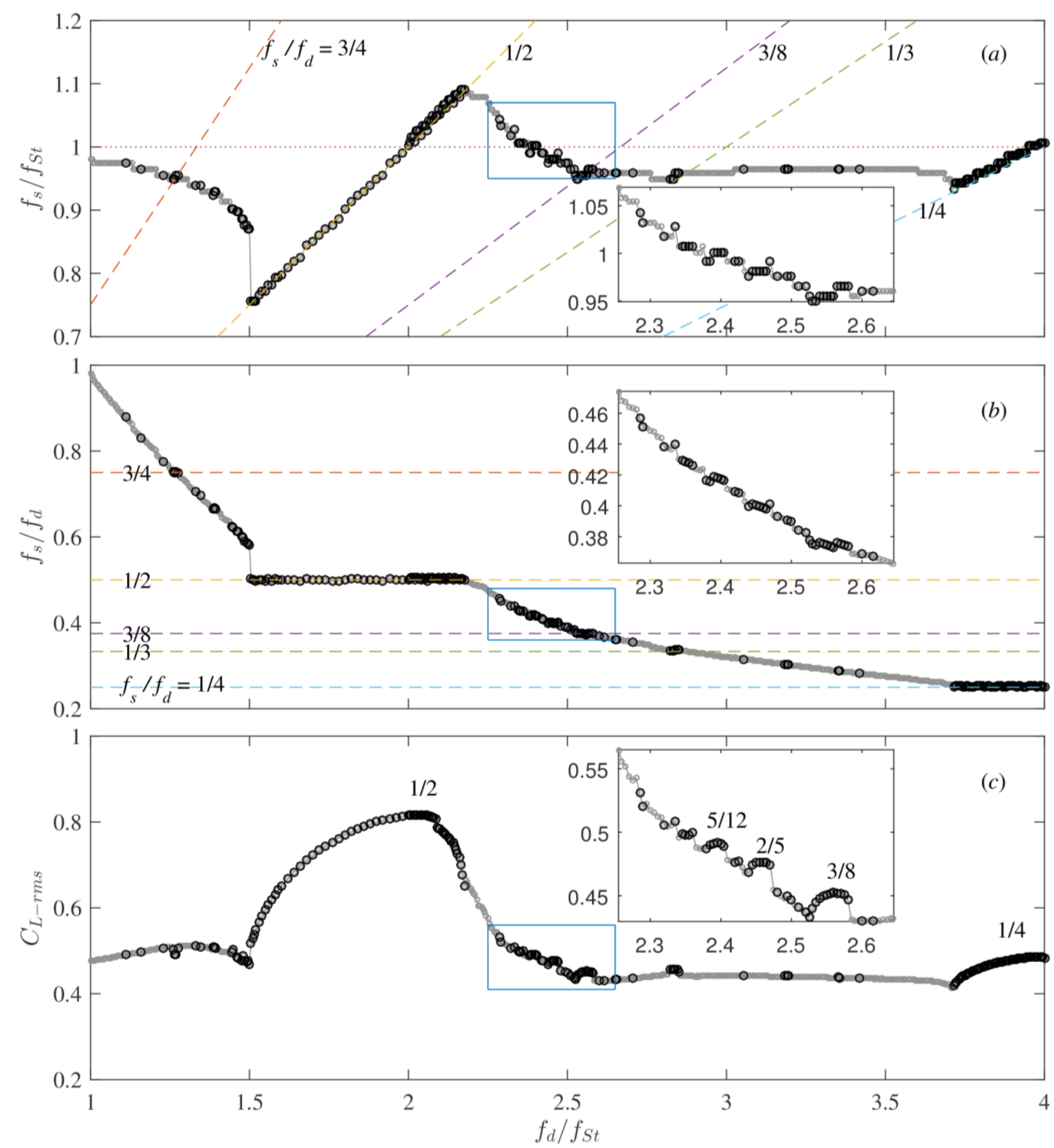

Figure 14 The change of vortex shedding frequency $\left(f_{s}\right)$ as a function of driving frequency $\left(f_{d}\right)$ at $A / D=0.1(a, b)$, along with the corresponding root-mean-square (rms) of the lift coefficient, $(c)$. Grey $\circ$ give all the simulated cases, while slightly larger black $\circ$ are the cases deemed synchronisation. A zoom-in area among $f_{d} / f_{S t}=2.25 \sim 2.65$ has been given on each panel. Dashed lines in $(a, b)$ show the reference $f_{s} / f_{d}$ values, whereas selected mode ratios are indicated in $(c)$ just above the region of synchronisation.

Figure 14(c) illustrates the variation in amplitudes of the lift coefficient with changes in $f_{d} / f_{S t}$. Large increases in the root-mean-square value of the lift coefficient $\left(C_{L-r m s}\right)$ were noticed in the 
synchronised regions, especially when the synchronisation tongue is wide (see the 1/2 and 1/4 modes). This is because as the separated flow becomes resonant to $f_{d}$, it is associated with the coalescence of small vortices and thus a more organised wake flow, causing an enhanced shear layer and a stronger entrainment wake (Wu et al. 1998). It is noticed in the zoomed inset of Figure 14(c) that responses of $C_{L-r m s}$ in the narrow synchronisation regions exhibit self-similarity to the wide regions. This is observed in each of the 5/12, 2/5, 3/8 modes as well as the $1 / 4$ synchronised region, which brings a local rise in $C_{L-r m s}$, holding an extraordinary resemblance to that of the $1 / 2$ mode. The self-similarity of $C_{L-r m s}$ values in different synchronisation modes suggests they share the same physical mechanism, while the magnitude of increase in $C_{L-r m s}$ at each mode region is consistent with the strength of frequency adjustment.

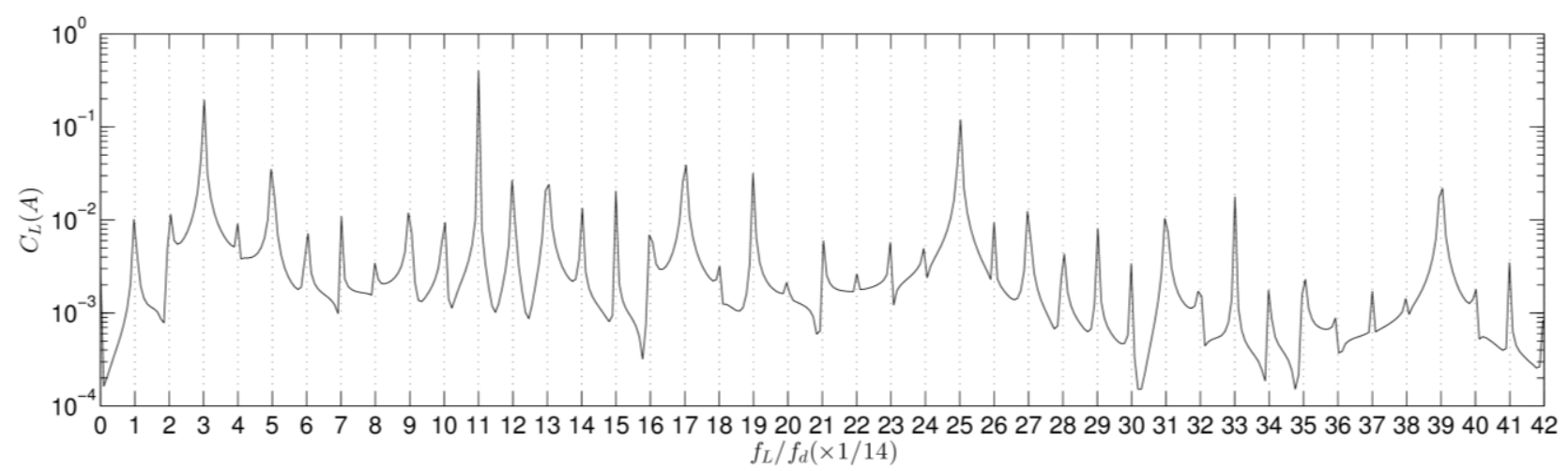

Figure 15 Locations of peaks in frequency domain for the $11 / 14$ mode at $\left(f_{d} / f_{S t}, A / D\right)=(1.0,0.305)$. The frequency resolution in $f_{L}$ is $1 / 750$.

\begin{tabular}{ccc}
\hline$L(p)$ & $(M, N)$ & Note \\
& & \\
$3 / 14$ & $(-1,1)$ & $-f_{s}+f_{d}$, major peak \\
$5 / 14$ & $(3,-2)$ & $3 f_{s}-2 f_{d}$, major peak \\
$11 / 14$ & $(1,0)$ & $f_{s}$, the strongest peak \\
$14 / 14$ & $(0,1)$ & $f_{d}$, minor peak \\
$25 / 14$ & $(1,1)$ & $f_{s}+f_{d}$, major peak \\
$39 / 14$ & $(1,2)$ & $f_{s}+2 f_{d}$, major peak \\
Table 3 Values of $(M, N)$ for locations of selected peaks in the frequency spectra in Figure 15
\end{tabular}

\subsection{Frequency peaks}

It has been shown the frequency content in the lift force can be very rich when the flow is quasiperiodic (Baek \& Sung 2000; Leontini et al. 2011), where nonlinear interactions between the driving and vortex shedding create relatively widespread peaks. In contrast, the spectra of synchronised cases exhibit only a number of sharply defined peaks, as observed from all locked-on spectra shown above, in consistent with previously experimental observations (Cetiner \& Rockwell 2001). The physical meaning of these peaks is the sum and difference of the $f_{s}$ and $f_{d}$. This phenomenon can be interpreted as when the nonlinear effect is less dominant as compared to the quasi-periodic case, $f_{s}$ is linearly dependent on $f_{d}$. The local peak locations $L(p)$ in the frequency content are therefore simply the sum and difference of the mode ratio $p / q$ combined with a pair of integer numbers $(M, N)$, formulated as

$$
L(p) / f_{d}=\left| \pm M \frac{p}{q} \pm N\right|=\left|\frac{ \pm M p \pm N q}{q}\right|,
$$


Or, in other words, the super harmonics of $1 / q$. By timing $f_{d}$ at both sides, this equation mathematically corresponds to all peaks at

$$
L(p)=\left| \pm M f_{s} \pm N f_{d}\right| .
$$

Further demonstration is shown in Figure 15 for a case with great complexity at $p / q=11 / 14$ (see Figure $11(c)$ for $C_{L}$ in the time domain). It is shown that all 42 peaks in the range of $f_{L} / f_{d} \in[0,3]$, strong or weak, can be represented by the super harmonics of $1 / 14$. The $(M, N)$ values of selected peaks are given in Table 3 (please note that there are more than one pair of $M$ and $N$ for these peaks).

\section{Conclusions}

Numerical simulations have been carried out in seeking the modes of synchronisation between the vortex shedding frequency $\left(f_{s}\right)$ and the driving frequency $\left(f_{d}\right)$ in the wake of a streamwise oscillatory cylinder at a fixed Reynolds number, $R e=175$ (based on the free stream velocity). The synchronisation is characterised by exactly $p$ pairs of vortices being shed over $q$ periods of cylinder oscillation, thus $f_{s}$ is detuned to a rational ratio of $f_{d}$, i.e. $f_{s} / f_{d}=p / q$ ( $p$ and $q$ are natural numbers, taken as $0,1,2,3,4, \ldots)$. This feature has given rise to a repeated transverse force every $q$ oscillation cycles and a certain type of spatial-temporal symmetric-global wake. The main conclusions are drawn as follows:

1) It is shown that the outcome of the competition between vortex formation induced by the steady flow and that by the cylinder oscillation leads to a relatively complete series of synchronisation modes. The mode ratios constitute the Farey sequence, including the primary synchronisation mode where $f_{s} / f_{d}=1 / 2$ (Tanida et al. 1973), and the nonlinear $P_{N}$ synchronisation mode where $f_{s} / f_{d}=(N-1) / N$ and $N$ is an integer (Leontini et al. 2013).

2) Although it is demonstrated that the synchronisation mode ratio $f_{s} / f_{d}$ can be almost any completely reduced rational ratio, $p / q$ (as can be seen for $p$ and $q \leq 10$ ), the synchronised flow field is relatively robust to the change of controlling parameters for small numbers (such as $1 / 2,1 / 4$, as well as $0 / 1$ ). That is, the synchronised flow favours ratios composed by small natural numbers.

3) The synchronised flow manifests itself in a variety of modes also preferring an even number as the denominator in the mode ratio to an odd one. This is attributed to the natural distribution of the Kármán vortex street. It can be interpreted as that both the background steady flow and the oscillatory perturbation control the wake, but the steady flow is the dominant mechanism in the present range of parameters.

4) When an odd number is chosen as the denominator in the mode ratio, however, the wake flow generally leans to one side of the axis of oscillation and the mean transverse force is not zero. This feature resembles that of the asymmetric vortex formation around oscillatory bodies without the steady flow (Tatsuno \& Bearman 1990; Tong et al. 2017). The region of mode ratio with an odd denominator is generally narrow, and the flow is sensitive to a change of driving frequency.

5) Whenever the wake flow is synchronised with the movement of the cylinder, $f_{s}$ is linearly dependent on the change of $f_{d}$, so the peaks in the Fourier spectrum of the transverse force is simply the sum and difference of $f_{s}$ and $f_{d}$. Since $f_{s}$ coincides with a rational ratio of $f_{d}$, it is demonstrated that the spectrum peaks are located at the super harmonics of $1 / q$. A nonlinear effect, namely the competition between the vortex shedding and cylinder oscillation, takes place for desynchronised cases and introduces less regular frequency content in the Fourier spectrum. 


\section{Acknowledgements}

This work was supported by the program from NSFC of China with Grant Nos. 51409035, 51479025 and 51490673. The financial support from China Postdoctoral Science Foundation with Grant No. 2014M551089 is acknowledged. The second and third authors would like to acknowledge the support from Australian Research Council through Grants DP110105171 and DP130103619. This work was also supported by resources provided by the Pawsey Supercomputing Centre with funding from the Australian Government and the Government of Western Australia. We are grateful to the referees for their constructive inputs.

\section{REFERENCES}

AL-MDALLAL, Q., LAWRENCE, K. \& KOCABIYIK, S. 2007. Forced streamwise oscillations of a circular cylinder: Locked-on modes and resulting fluid forces. J. Fluids Struct., 23, 681-701.

ANAGNOSTOPOULOS, P. 2000. Numerical study of the flow past a cylinder excited transversely to the incident stream. Part 1: Lock-in zone, hydrodynamic forces and wake geometry. J. Fluids Struct., 14, 819-851.

BAEK, S.-J. \& SUNG, H. J. 2000. Quasi-periodicity in the wake of a rotationally oscillating cylinder. J. Fluid Mech., 408, 275-300.

BARBI, C., FAVIER, D., MARESCA, C. \& TELIONIS, D. 1986. Vortex shedding and lock-on of a circular cylinder in oscillatory flow. J. Fluid Mech., 170, 527-544.

BISHOP, R. \& HASSAN, A. 1964. The lift and drag forces on a circular cylinder oscillating in a flowing fluid. Proc. $R$. Soc. A, 277, 51-75.

BLACKBURN, H. M. \& HENDERSON, R. D. 1999. A study of two-dimensional flow past an oscillating cylinder. $J$. Fluid Mech., 385, 255-286.

CANTWELl, C., MOXEY, D., COMERFORD, A., BOLIS, A., ROCCO, G., MENGALDO, G., DE GRAZIA, D., YAKOVLEV, S., LOMBARD, J.-E. \& EKELSCHOT, D. 2015. Nektar++: An open-source spectral/hp element framework. Comput. Phys. Commun., 192, 205-219.

CARBERRY, J., SHERIDAN, J. \& ROCKWELL, D. 2005. Controlled oscillations of a cylinder: forces and wake modes. J. Fluid Mech., 538, 31-69.

CETINER, O. \& ROCKWELL, D. 2001. Streamwise oscillations of a cylinder in a steady current. Part 1. Locked-on states of vortex formation and loading. J. Fluid Mech., 427, 1-28.

D'ADAMO, J., GODOY-DIANA, R. \& WESFREID, J. E. 2011. Spatiotemporal spectral analysis of a forced cylinder wake. Phys. Rev. E, 84, 056308.

DETEMPLE-LAAKE, E. \& ECKELMANN, H. 1989. Phenomenology of Kármán vortex streets in oscillatory flow. Exp. Fluids, 7, 217-227.

GRIFFIN, O. M. \& HALL, M. 1991. Review_vortex shedding lock-on and flow control in bluff body wakes. J. Fluids Eng., 113, 526-537.

GRIFFIN, O. M. \& RAMBERG, S. E. 1974. The vortex-street wakes of vibrating cylinders. J. Fluid Mech., 66, 553-576.

GRIFFIN, O. M. \& RAMBERG, S. E. 1976. Vortex shedding from a cylinder vibrating in line with an incident uniform flow. J. Fluid Mech., 75, 257-271.

JIANG, H., CHENG, L., DRAPER, S., AN, H. \& TONG, F. 2016. Three-dimensional direct numerical simulation of wake transitions of a circular cylinder. J. Fluid Mech., 801, 353-391.

KARNIADAKIS, G. E., ISRAELI, M. \& ORSZAG, S. A. 1991. High-order splitting methods for the incompressible Navier-Stokes equations. J. Comput. Phys., 97, 414-443.

KARNIADAKIS, G. E. \& TRIANTAFYLLOU, G. S. 1989. Frequency selection and asymptotic states in laminar wakes. J. Fluid Mech., 199, 441-469.

KONSTANTINIDIS, E. \& BALABANI, S. 2007. Symmetric vortex shedding in the near wake of a circular cylinder due to streamwise perturbations. J. Fluids Struct., 23, 1047-1063.

KONSTANTINIDIS, E. \& BOURIS, D. 2016. Vortex synchronization in the cylinder wake due to harmonic and nonharmonic perturbations. J. Fluid Mech., 804, 248-277.

KOOPMANN, G. 1967. The vortex wakes of vibrating cylinders at low Reynolds numbers. J. Fluid Mech., 28, $501-512$.

LEONTINI, J., STEWART, B., THOMPSON, M. \& HOURIGAN, K. 2006. Wake state and energy transitions of an oscillating cylinder at low Reynolds number. Phys. Fluids, 18, 067101.

LEONTINI, J. S., LO JACONO, D. \& THOMPSON, M. C. 2011. A numerical study of an inline oscillating cylinder in a free stream. J. Fluid Mech., 688, 551-568.

LEONTINI, J. S., LO JACONO, D. \& THOMPSON, M. C. 2013. Wake states and frequency selection of a streamwise oscillating cylinder. J. Fluid Mech., 730, 162-192.

LOTFY, A. \& ROCKWELL, D. 1993. The near-wake of an oscillating trailing edge: mechanisms of periodic and aperiodic response. J. Fluid Mech., 251, 173-201. 
MARZOUK, O. A. \& NAYFEH, A. H. 2009. Reduction of the loads on a cylinder undergoing harmonic in-line motion. Phys. Fluids, 21, 083103.

MITTAL, S., RATNER, A., HASTREITER, D. \& TEZDUYAR, T. E. 1991. Space-time finite element computation of incompressible flows with emphasis on flows involving oscillating cylinders. International Video Journal of Engineering Research, 1, 83-96.

MORSE, T. \& WILLIAMSON, C. 2009. Prediction of vortex-induced vibration response by employing controlled motion. J. Fluid Mech., 634, 5-39.

NAZARINIA, M., LO JACONO, D., THOMPSON, M. C. \& SHERIDAN, J. 2012. Flow over a cylinder subjected to combined translational and rotational oscillations. J. Fluids Struct., 32, 135-145.

NEWMAN, D. J. \& KARNIADAKIS, G. E. 1997. A direct numerical simulation study of flow past a freely vibrating cable. J. Fluid Mech., 344, 95-136.

OLINGER, D. \& SREENIVASAN, K. 1988. Nonlinear dynamics of the wake of an oscillating cylinder. Phys. Rev. Lett., 60, 797.

OLINGER, D. J. 1993. A low - dimensional model for chaos in open fluid flows. Phys. Fluids, 5, 1947-1951.

OLINGER, D. J. 1998. A low-order model for vortex shedding patterns behind vibrating flexible cables. Phys. Fluids, 10, 1953-1961.

ONGOREN, A. \& ROCKWELL, D. 1988a. Flow structure from an oscillating cylinder Part 1. Mechanisms of phase shift and recovery in the near wake. J. Fluid Mech., 191, 197-223.

ONGOREN, A. \& ROCKWELL, D. 1988b. Flow structure from an oscillating cylinder. Part 2: Mode competition in the near wake. J. Fluid Mech., 191, 225-245.

OTT, E. 2002. Chaos in dynamical systems, Cambridge university press.

RAO, A., LEONTINI, J., THOMPSON, M. \& HOURIGAN, K. 2013. Three-dimensionality in the wake of a rotating cylinder in a uniform flow. J. Fluid Mech., 717, 1-29.

SARPKAYA, T., BAKMIS, C. \& STORM, M. 1984. Hydrodynamic forces from combined wave and current flow on smooth and rough circular cylinders at high Reynolds numbers. Offshore Technology Conference. Houston, TX.

STANSBY, P. 1976. The locking-on of vortex shedding due to the cross-stream vibration of circular cylinders in uniform and shear flows. J. Fluid Mech., 74, 641-665.

TANG, G., CHENG, L., LU, L., ZHAO, M., TONG, F. \& DONG, G. 2016. Vortex formation in the wake of a streamwisely oscillating cylinder in steady flow. In: ZHOU, Y., LUCEY, A., LIU, Y. \& HUANG, L. (eds.) Fluid-Structure-Sound Interactions and Control. Springer, Berlin, Heidelberg.

TANIDA, Y., OKAJIMA, A. \& WATANABE, Y. 1973. Stability of a circular cylinder oscillating in uniform flow or in a wake. J. Fluid Mech., 61, 769-784.

TATSUNO, M. 1972. Vortex streets behind a circular cylinder oscillating in the direction of flow. Bull. Res. Inst. Appl. Mech. Kyushu Univ, 36, 25-37.

TATSUNO, M. \& BEARMAN, P. 1990. A visual study of the flow around an oscillating circular cylinder at low Keulegan-Carpenter numbers and low Stokes numbers. J. Fluid Mech., 211, 157-182.

TONG, F., CHENG, L., XIONG, C., DRAPER, S., AN, H. \& LOU, X. 2017. Flow regimes for a square cross-section cylinder in oscillatory flow. J. Fluid Mech., 813, 85-109.

TONG, F., CHENG, L., ZHAO, M. \& AN, H. 2015. Oscillatory flow regimes around four cylinders in a square arrangement under small KC and Re conditions. J. Fluid Mech., 769, 298-336.

TUDBALL-SMITH, D., LEONTINI, J. S., SHERIDAN, J. \& LO JACONO, D. 2012. Streamwise forced oscillations of circular and square cylinders. Phys. Fluids, 24, 111703.

WILLIAMSON, C. \& ROSHKO, A. 1988. Vortex formation in the wake of an oscillating cylinder. J. Fluids Struct., 2, 355-381.

WILLIAMSON, C. H. K. \& BROWN, G. L. 1998. A series in 1/ $\sqrt{ }$ Re to represent the Strouhal-Reynolds number relationship of the cylinder wake. J. Fluids Struct., 12, 1073-1085.

WOO, H. 1999. A note on phase-locked states at low Reynolds numbers. J. Fluids Struct., 13, 153-158.

WU, J.-Z., LU, X.-Y., DENNY, A. G., FAN, M. \& WU, J.-M. 1998. Post-stall flow control on an airfoil by local unsteady forcing. J. Fluid Mech., 371, 21-58.

XU, S., ZHOU, Y. \& WANG, M. 2006. A symmetric binary-vortex street behind a longitudinally oscillating cylinder. $J$. Fluid Mech., 556, 27-43.

ZDRAVKOVICH, M. 1996. Different modes of vortex shedding: an overview. J. Fluids Struct., 10, 427-437. 